\title{
Doppler-Hitchhiker: A Novel Passive Synthetic Aperture Radar Using Ultranarrowband Sources of Opportunity
}

\author{
Ling Wang, Member, IEEE, Can Evren Yarman, and Birsen Yazıcı, Senior Member, IEEE
}

\begin{abstract}
In this paper, we present a novel synthetic aperture radar imaging modality that uses ultranarrowband sources of opportunity and passive airborne receivers to form an image of the ground. Due to its combined passive synthetic aperture and high Doppler resolution of the transmitted waveforms, we refer to this modality as the Doppler Synthetic Aperture Hitchhiker or Doppler-hitchhiker for short. Our imaging method first correlates the windowed signal obtained from one receiver with the scaled and translated version of the received signal in another window from the same or another receiver. We show that this correlation processing removes the transmitter-related variables from the phase of the resulting operator that maps the radiance of the scene to the correlated signals. We define a concept of passive Doppler scale factor using the radial velocities of the receivers. Next, we show that the scaled, translated, and correlated signal is the projection of the scene radiance onto the contours that are formed by the intersection of the surfaces of constant passive Doppler scale factor and ground topography. We use microlocal analysis to design a generalized filtered-backprojection operator to reconstruct the scene radiance from its projections. Our analysis shows that the resolution of the reconstructed images improves with the increased time duration and center frequency of the transmitted ultranarrowband signals. Our reconstruction method is analytic and therefore can be made computationally efficient. Furthermore, it easily accommodates arbitrary flight trajectories, nonflat topography, and system-related parameters. We present numerical simulations to demonstrate the performance of our imaging method.
\end{abstract}

Index Terms-Filtered-backprojection (FBP), passive imaging, passive radar, passive synthetic aperture radar, ultra-narrowband waveforms.

Manuscript received October 22, 2010; revised January 31, 2011; accepted March 2, 2011. Date of publication June 8, 2011; date of current version September 28, 2011. This work was supported in part by the Air Force Office of Scientific Research (AFOSR) under Agreements FA9550-07-1-0363 and FA9550-09-1-0013 and in part by the National Science Foundation (NSF) under Grant CCF-08030672. The views and conclusions contained herein are those of the authors and should not be interpreted as necessarily representing the official policies or endorsements, either expressed or implied, of the Air Force Research Laboratory or the U.S. Government. The U.S. Government is authorized to reproduce and distribute reprints for Governmental purposes notwithstanding any copyright notation thereon.

L. Wang is with the Department of Information and Communication Engineering, Nanjing University of Aeronautics and Astronautics, Nanjing 210016, China (e-mail: wanglrpi@gmail.com).

C. E. Yarman is with Houston Technology Center, WesternGecoSchlumberger, Houston, TX 77042 USA (e-mail: yarman@ecse.rpi.edu).

B. Yazicı is with the Department of Electrical, Computer, and Systems Engineering, Rensselaer Polytechnic Institute, Troy, NY 12180 USA (e-mail: B. Y: yazici@ecse.rpi.edu).

Color versions of one or more of the figures in this letter are available online at http://ieeexplore.ieee.org.

Digital Object Identifier 10.1109/TGRS.2011.2142420

\section{INTRODUCTION}

$\mathbf{I}$ $\mathrm{N}$ RECENT years, the presence of ambient radio frequencies due to a growing number of transmitters of opportunity, such as radio, television, and cell phone stations, has motivated interest in passive radar applications [1]-[21].

While many passive radar applications are focused on the detection of targets with ground-based receivers [1]-[16], a number of methods for passive synthetic aperture radar (SAR) have been developed [17]-[19]. The methods presented in [1]-[18] require either the knowledge of the transmitter locations and transmitted waveforms or receivers with high directivity that are within the beams of the transmitter antennas. The direct line-ofsight to the transmitters is then used to estimate the transmitted waveforms and the transmitter location to perform matched filtering. In [19], we reported a novel passive synthetic aperture imaging method based on the spatiotemporal correlation of the received signal and the filtered-backprojection technique. Unlike the methods in [1]-[18], this method does not require receivers with high directivity or a priori knowledge about the transmitter locations and transmitted waveforms. The resolution analysis of the method shows that it is suitable for high-range-resolution waveforms, such as wideband pulses. However, most of the transmitters of opportunity, such as radio and television stations, transmit single-frequency or ultranarrowband waveforms. In this paper, we present a new passive synthetic aperture imaging modality using sources of opportunity transmitting single-frequency or ultranarrowband waveforms. These waveforms are also referred to as highDoppler-resolution or continuous-wave (CW) waveforms. Thus, we refer to the resulting modality as the Doppler Synthetic Aperture Hitchhiker (DSAH) or "Doppler-hitchhiker" for short.

Fig. 1 shows the DSAH imaging geometry along with the imaging process. There are three key interrelated ideas in our passive ultranarrowband synthetic aperture imaging method. The first two are related to the development of a novel forward model, and the last one is related to the inversion of this model for image formation. Our objective is to develop a passive forward model so that the phase of the model does not involve transmitter related terms and allows us to determine the location of the scatterers on the ground for which the moving receivers observe a constant passive Doppler scaling factor; we use microlocal techniques to recover the radiance of scatterers for which the passive Doppler scaling factor is constant. For each pair of receivers, our method first correlates 


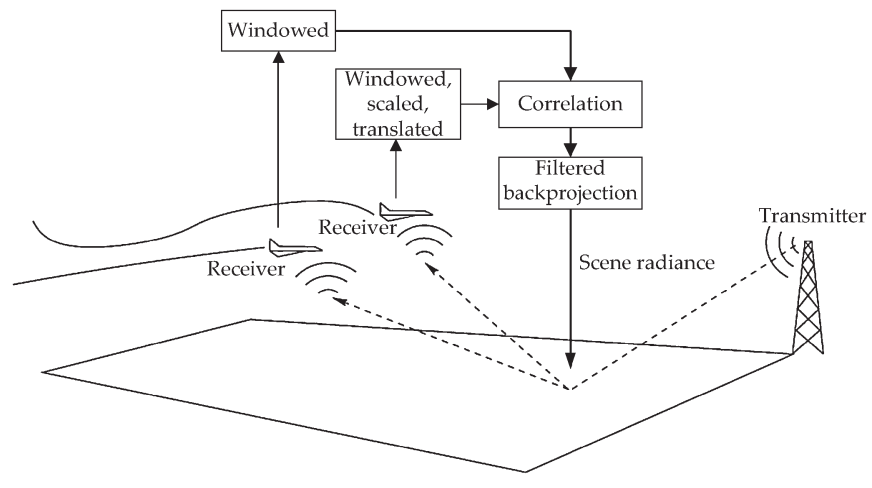

Fig. 1. Illustration of the DSAH imaging geometry along with the imaging process.

the windowed signal obtained from one of the receivers with the windowed, scaled, and translated version of the received signal from another receiver. If only a single receiver is available, the received signal is windowed, scaled, translated, and correlated with itself. The scaling process facilitates imaging of scatterers on the DSAH iso-Doppler contours that we introduce in this paper. The correlation processing removes the transmitterrelated terms from the phase component of the resulting forward model that relates the scene radiance to the correlated data. This means that the correlated data can be backpropagated to the scene radiance without knowing the location of the transmitters. We next introduce the concept of passive Doppler scale factor using the radial velocities of the receivers [21]. In the context of Doppler-hitchhiker, we refer to the passive Doppler scale factor as the Doppler-hitchhiker scale factor. Note that, under the slow-mover assumption, the Dopplerhitchhiker scale factor is equivalent to the hitchhiker-Doppler that we introduced in [19]. We define the points on the ground topography at which the Doppler-hitchhiker scale factor is constant, as the DSAH iso-Doppler contour. Under slow-mover assumption, DSAH iso-Doppler contours are approximated by the hitchhiker iso-Doppler contours that we introduced in [19]. The high-frequency analysis of the forward model shows that the windowed scaled-and-translated correlation of the received signals is the projection of the scene radiance onto the hitchhiker iso-Doppler contours. Our analysis shows that the spread of the hitchhiker iso-Doppler contours on the ground is directly related to the Doppler ambiguity of the transmitted waveforms. Since the Doppler ambiguity of CW or ultranarrowband waveforms is very good, the resulting contours on the ground are narrow. Thus, the backpropagation of the scaled, translated, and correlated data onto the hitchhiker iso-Doppler contours results in high-resolution passive SAR imaging using ultranarrowband signals. We use microlocal techniques to develop a filteredbackprojection (FBP) reconstruction of the scene radiance. The analysis of the point spread function (PSF) of the imaging operator shows that the image is reconstructed at the intersection of the hitchhiker iso-Doppler and hitchhiker iso-Dopplerrate contours introduced in this paper. The final image of the scene radiance is formed by the superposition of the images obtained for each pair of receivers at each time translation over a range of time translations. The resolution analysis of our imaging method shows that the resolution improves with the higher frequency and longer time duration of the transmitted ultranarrowband signals.
Doppler-hitchhiker has many advantages: To the best of our knowledge, Doppler-hitchhiker is the first fully passive SAR modality that uses ultranarrowband waveforms of opportunity. Doppler-hitchhiker can be used with stationary and/or mobile, cooperative and noncooperative sources of opportunity. Unlike the existing passive radar detection systems [1]-[18], Dopplerhitchhiker does not require receivers with high directivity and can be used under nonideal imaging scenarios such as arbitrary flight trajectories and nonflat topography. The image reconstruction method has the desirable property of preserving the visible edges of the scene radiance. Additionally, it is an analytic image formation method that can be made computationally efficient using fast-backprojection methods [22].

The organization of this paper is as follows. In Section II, we present the windowed scaled-and-translated correlation of the received measurements and determine the forward model that relates the scene radiance to correlated measurements. We also present the high-frequency analysis of the Doppler-hitchhiker forward model. In Section III, we develop a filtered-backprojection-type image formation method for Doppler-hitchhiker and analyze the underlying geometry and resolution of the Doppler-hitchhiker image formation. In Section V, we present numerical simulations to verify the theoretical results and demonstrate the performance of the image formation method. Finally, in Section VI, we summarize our results and conclude our discussion. This paper includes two appendices presenting the derivation of the intermediate results needed for the image reconstruction method.

We use the following notational conventions throughout this paper. The bold Roman, bold italic, and Roman lower-case letters are used to denote variables in $\mathbb{R}^{3}, \mathbb{R}^{2}$, and $\mathbb{R}$, respectively, i.e., $\mathbf{z}=(\boldsymbol{z}, z) \in \mathbb{R}^{3}$, with $z \in \mathbb{R}^{2}$ and $z \in \mathbb{R}$. The calligraphic letters $(\mathcal{F}, \mathcal{K}$, etc. $)$ are used to denote operators. Table I lists the notation used throughout this paper.

\section{Doppler-HitchhiKer MeAsurement Model}

In this section, we first describe the received signal model for a given transmitter transmitting a CW signal and next derive a novel forward model by correlating the windowed, scaled, and translated received signal. The resulting processed data are then used to form an image of the scene radiance by a filteredbackprojection method.

\section{A. Model for the Received Signal}

Given a pair of transmitter and receiver antennas located at $\mathbf{T}$ and $\mathbf{R}$, respectively, we model the received signal by [23]

$$
\begin{aligned}
f(t, \mathbf{R}, \mathbf{T}) & \approx \int \frac{e^{i \omega\left(t-(|\mathbf{R}-\mathbf{z}|+|\mathbf{z}-\mathbf{T}|) / c_{0}\right)}}{(4 \pi)^{2}|\mathbf{R}-\mathbf{z}||\mathbf{z}-\mathbf{T}|} \omega^{2} \hat{p}(\omega) \\
& \times J_{\operatorname{tr}}(\omega, \widehat{\mathbf{z}-\mathbf{T}}, \mathbf{T}) J_{\mathrm{rc}}(\omega, \widehat{\mathbf{z}-\mathbf{R}}, \mathbf{R}) V(\mathbf{z}) d \omega d \mathbf{z}
\end{aligned}
$$

where $t$ denotes time, $c_{0}$ denotes the speed of light in free space, $V(\mathbf{z})$ is the reflectivity function, $\hat{p}$ denotes the Fourier transform of the transmitted waveform $p(t)$, and $J_{\mathrm{tr}}$ and $J_{\mathrm{rc}}$ are the transmitter and receiver antenna beam patterns, respectively. 
TABLE I

TABLE OF NOTATIONS

\begin{tabular}{|c|c|}
\hline$\overline{\text { Symbol }}$ & Designation \\
\hline $\mathbf{T}$ & Transmitter location \\
\hline $\mathbf{R}$ & Receiver location \\
\hline$c_{0}$ & Speed of light in free space \\
\hline$\omega_{0}$ & $\begin{array}{l}\text { Carrier frequency of the transmit- } \\
\text { ted waveform }\end{array}$ \\
\hline$t$ & Time variable \\
\hline$f(t, \mathbf{R}, \mathbf{T})$ & $\begin{array}{l}\text { Received signal at } \mathbf{R} \text { due to a } \\
\text { transmitter at } \mathbf{T}\end{array}$ \\
\hline $\mathbf{z}=(\boldsymbol{z}, \boldsymbol{\psi}(\boldsymbol{z}))$ & Earth's surface \\
\hline$V(\mathbf{z})$ & Reflectivity function \\
\hline$\rho(z)$ & Surface reflectivity \\
\hline$\delta(z)$ & Dirac delta function \\
\hline$\widehat{\mathbf{z}}$ & $\begin{array}{l}\text { Unit vector in the direction of } \mathbf{z} \in \\
\mathbb{R}^{3}\end{array}$ \\
\hline$\gamma_{i}(t)$ & $\begin{array}{l}\text { Flight trajectory of the } i \text { th re- } \\
\text { ceiver }\end{array}$ \\
\hline$s_{i}(t)=f\left(t, \gamma_{i}(t), \mathbf{T}\right)$ & $\begin{array}{l}\text { Signal collected along the re- } \\
\text { ceiver trajectory } \gamma_{i}(t) \text { due to a } \\
\text { transmitter located at } \mathbf{T}\end{array}$ \\
\hline$\tau, \tau^{\prime}$ & Variables for temporal translation \\
\hline & Temporal scaling factor \\
\hline$\phi(t)$ & Temporal windowing function \\
\hline$Q_{i j}\left(\boldsymbol{z}, t, \tau^{\prime}, \tau\right)$ & Reconstruction filter \\
\hline$c_{i j}\left(\tau^{\prime}, \tau, \mu\right)$ & $\begin{array}{l}\text { Windowed, scaled-and-translated } \\
\text { correlations of the received sig- } \\
\text { nals from the } i \text { th and } j \text { th receiver } \\
\text { trajectories }\end{array}$ \\
\hline$R_{\rho}(\boldsymbol{z})$ & Scene radiance \\
\hline$R_{T}\left(\omega_{0}, \boldsymbol{z}, \boldsymbol{z}^{\prime}, \mathbf{T}\right)$ & Transmitter irradiance \\
\hline$A_{R_{i j}}\left(\omega_{0}, \boldsymbol{z}, \boldsymbol{z}^{\prime}, t, \tau^{\prime}, \tau, \mu\right)$ & $\begin{array}{l}\text { Product of the receiver antenna } \\
\text { beam patterns }\end{array}$ \\
\hline$G_{i j}\left(\mathbf{z}, \mathbf{z}^{\prime}, t, \tau^{\prime}, \tau, \mu\right)$ & $\begin{array}{l}\text { Product of the geometric spread- } \\
\text { ing factors due to transmitter } \mathbf{T} \\
\text { and receivers } i \text { and } j\end{array}$ \\
\hline & Forward modeling operator \\
\hline$\varphi_{i j}\left(t, \boldsymbol{z}, \tau^{\prime}, \tau, \mu\right)$ & Phase of the operator $\mathcal{F}_{i j}$ \\
\hline$A_{i j}\left(\boldsymbol{z}, t, \tau^{\prime}, \tau, \mu\right)$ & Amplitude of the operator $\mathcal{F}_{i j}$ \\
\hline$S_{i j}\left(\tau^{\prime}, \tau, \mathbf{z}\right)$ & Doppler-hitchhiker scale factor \\
\hline$F_{i j}\left(\tau^{\prime}, \tau, \mu\right)$ & $\begin{array}{l}\text { Hitchhiker (DSAH) iso-Doppler } \\
\text { contour }\end{array}$ \\
\hline$\dot{F}_{j}(\tau, C)$ & $\begin{array}{l}\text { Hitchhiker (DSAH) iso-Doppler- } \\
\text { rate contour }\end{array}$ \\
\hline$\widetilde{R}_{\rho}(\boldsymbol{z})$ & Image of the scene radiance \\
\hline & Filtered-backprojection operator \\
\hline$L\left(\boldsymbol{z}, \boldsymbol{z}^{\prime}\right)$ & Point spread function \\
\hline$\Omega_{i j, \tau^{\prime}, z}$ & $\begin{array}{l}\text { Partial data collection manifold at } \\
\left(\tau^{\prime}, \boldsymbol{z}\right) \text { due to the } i \text { th and } j \text { th } \\
\text { receivers }\end{array}$ \\
\hline$\Omega_{z}$ & $\begin{array}{l}\text { Data collection manifold at } \mathbf{z} \in \\
\mathbb{R}^{3}\end{array}$ \\
\hline$L_{\phi}$ & Length of the support of $\phi(t)$ \\
\hline
\end{tabular}

We make the assumption that the Earth's surface is located at a position given by $\mathbf{z}=(\boldsymbol{z}, \boldsymbol{\psi}(\boldsymbol{z})) \in \mathbb{R}^{3}$, where $\boldsymbol{z} \in \mathbb{R}^{2}$ and $\psi: \mathbb{R}^{2} \rightarrow \mathbb{R}$ is a known function for the ground topography. Furthermore, we assume that the scattering takes place in a thin region near the surface. Thus, the reflectivity function is in the form

$$
V(\mathbf{z})=\rho(\boldsymbol{z}) \delta(z-\boldsymbol{\psi}(\boldsymbol{z})) .
$$

For a narrowband waveform, we have

$$
p(t)=e^{i \omega_{0} t} \tilde{p}(t)
$$

where $\omega_{0}$ denotes the carrier frequency and $\tilde{p}(t)$ is the complex envelope of $p$, which is slow varying as a function of $t$ compared to $e^{i \omega_{0} t}$.
Using (2) and the Fourier transform of (3) and under the assumption of broadband antenna, after algebraic rearrangements, (1) becomes

$$
\begin{aligned}
& f(t, \mathbf{R}, \mathbf{T}) \\
& \approx \int \frac{e^{i \omega_{0}\left(t-(|\mathbf{R}-\mathbf{z}|+|\mathbf{z}-\mathbf{T}|) / c_{0}\right)}}{(4 \pi)^{2}|\mathbf{R}-\mathbf{z}||\mathbf{z}-\mathbf{T}|} \\
& \times \tilde{p}\left(t-(|\mathbf{R}-\mathbf{z}|+|\mathbf{z}-\mathbf{T}|) / c_{0}\right) \\
& \times J_{\operatorname{tr}}\left(\omega_{0}, \widehat{\mathbf{z}-\mathbf{T}}, \mathbf{T}\right) J_{\mathrm{rc}}\left(\omega_{0}, \overline{\mathbf{z}-\mathbf{R}}, \mathbf{R}\right) \rho(\boldsymbol{z}) d \boldsymbol{z}
\end{aligned}
$$

where $\widehat{\mathbf{z}}=\mathbf{z} /|\mathbf{z}|$ denotes the unit vector in the direction of $\mathbf{z} \in$ $\mathbb{R}^{3}$. For the rest of this paper, unless otherwise stated, we use $\mathbf{z}=\mathbf{z}(\boldsymbol{z})=(\boldsymbol{z}, \boldsymbol{\psi}(\boldsymbol{z}))$.

Our imaging method is applicable to both mobile and stationary sources of opportunity. However, for the rest of our discussion, we assume that there is a single stationary transmitter of opportunity illuminating the scene. This allows us to simplify the analysis and distill the important aspects that can readily be generalized using the ideas similar to the ones presented in our work [19].

Let $\mathbf{T} \in \mathbb{R}^{3}$ denote the location of the transmitter of opportunity, and let there be $N$ airborne receivers, each traversing a smooth trajectory $\gamma_{i}(t), i=1, \ldots, N$.

We define

$$
s_{i}(t)=f\left(t, \gamma_{i}(t), \mathbf{T}\right)
$$

as the received signal by the $i$ th receiver, $i=1, \ldots, N$.

Note that, for multiple transmitters transmitting the same ultranarrowband waveform with different carrier frequencies, the signal received by the $i$ th receiver can be written as

$$
s_{i}(t)=\int f\left(t, \gamma_{i}(t), \mathbf{T}\right) d \mathbf{T} d \omega_{0}
$$

Since each transmitted frequency can be isolated with appropriate bandpass filtering, without loss of generality, we perform our analysis with respect to a fixed carrier frequency $\omega_{0}$.

\section{B. Windowed Scaled-and-Translated Correlation of Received Signals}

We now use the received signal model presented in the previous section to develop a forward model that performs the following: 1) It does not depend on transmitter locations, and 2) it facilitates imaging of scatterers for which the moving receivers observe a constant passive Doppler scaling factor. We start forming the forward model by first dividing the received signal at different (or same) receivers into different windows. We then scale, translate, and correlate the windowed received signal. The correlation process removes the transmitter-related terms from the phase of the resulting model. The scaling allows us to determine the location of the scatters on the stationary ground for which the moving receivers observe a constant Doppler scaling factor in certain directions. This processing of the received signal and the resulting forward model for passive synthetic aperture imaging with ultranarrowband waveforms are described in detail hereinafter.

We define the windowed scaled-and-translated correlation of the received signals $s_{i}$ and $s_{j}$ by

$$
c_{i j}\left(\tau^{\prime}, \tau, \mu\right)=\int s_{i}\left(t+\tau^{\prime}\right) s_{j}^{*}(\mu t+\tau) \phi(t) d t
$$


for some $\tau, \tau^{\prime} \in \mathbb{R}$ and $\mu \in \mathbb{R}^{+}, i, j=1, \ldots, N$, where $\phi(t)$ is a smooth compactly supported temporal windowing function centered at $t=0$ and ${ }^{*}$ denotes the complex conjugation. Note that, for a single receiver, we only have $c_{i j}=c_{11}$.

In the following sections, we develop a mapping that relates the expected value of the correlated measurements $c_{i j}$ to the scene to be imaged. We assume that the sources of opportunity are noncooperative where the location of the transmitter $\mathbf{T}$ and transmitter antenna beam pattern $J_{\text {tr }}$ are unknown. The result can be easily extended for the case of cooperative sources of opportunity where these quantities are assumed to be known. For both cases, the carrier frequency of the transmitted waveform $\omega_{0}$ is assumed to be known.

We use a stochastic model for the transmitter antenna beam pattern $J_{\mathrm{tr}}$ and the scene reflectivity $\rho$. Let $C_{\rho}$ and $C_{J_{\mathrm{tr}}}$ denote the correlation function of $\rho$ and $J_{\mathrm{tr}}$, respectively

$$
\begin{aligned}
C_{\rho}\left(\boldsymbol{z}, \boldsymbol{z}^{\prime}\right) & =E\left[\rho(\boldsymbol{z}) \rho^{*}\left(\boldsymbol{z}^{\prime}\right)\right] \\
C_{J_{\mathrm{tr}}}\left(\omega_{0}, \boldsymbol{z}, \boldsymbol{z}^{\prime}, \mathbf{T}\right) & =E\left[J_{\mathrm{tr}}\left(\omega_{0}, \widehat{\mathbf{z}-\mathbf{T}}, \mathbf{T}\right) J_{\mathrm{tr}}^{*}\left(\omega_{0}, \widehat{\mathbf{z}^{\prime}-\mathbf{T}}, \mathbf{T}\right)\right] .
\end{aligned}
$$

We assume that the scene reflectivity $\rho$ and the transmit antenna beam pattern $J_{\text {tr }}$ are statistically independent. Next, we make the incoherent field approximation [24] by assuming that $\rho$ and $J_{\mathrm{tr}}$ satisfy the following equalities:

$$
\begin{aligned}
C_{\rho}\left(\boldsymbol{z}, \boldsymbol{z}^{\prime}\right) & =R_{\rho}(\boldsymbol{z}) \delta\left(\boldsymbol{z}-\boldsymbol{z}^{\prime}\right) . \\
C_{J_{\mathrm{tr}}}\left(\omega_{0}, \boldsymbol{z}, \boldsymbol{z}^{\prime}, \mathbf{T}\right) & =R_{T}\left(\omega_{0}, \boldsymbol{z}, \boldsymbol{z}^{\prime}, \mathbf{T}\right) \delta\left(\boldsymbol{z}-\boldsymbol{z}^{\prime}\right) .
\end{aligned}
$$

Note that $R_{\rho}$ is the average power of the electromagnetic radiation emitted by the scene at location $z$ and $R_{T}$ is the average power of the electromagnetic radiation emitted by the transmitter at location $\mathbf{T}$ that is incident on the target surface at $z$. In this regard, $R_{\rho}$ is referred to as the scene radiance and $R_{T}$ is referred to as the transmitter irradiance [24].

Substituting $s_{i}$ and $s_{j}$ into $c_{i j}$, under the assumption that $\rho$, $J_{\mathrm{tr}}$, and $|\mathbf{T}-\mathbf{z}|$ are statistically independent, and using (10) and (11), we express the expectation of $c_{i j}$ as

$$
\begin{aligned}
E\left[c_{i j}\right. & \left.\left(\tau^{\prime}, \tau, \mu\right)\right] \\
= & \frac{\omega_{0}^{4}}{(4 \pi)^{4}} \int e^{i \omega_{0}\left(t+\tau^{\prime}-\left(\left|\boldsymbol{\gamma}_{i}\left(t+\tau^{\prime}\right)-\mathbf{z}\right|+|\mathbf{T}-\mathbf{z}|\right) / c_{0}\right)} \\
& \times e^{-i \omega_{0}\left(\mu t+\tau-\left(\left|\boldsymbol{\gamma}_{j}(\mu t+\tau)-\mathbf{z}^{\prime}\right|+\left|\mathbf{T}-\mathbf{z}^{\prime}\right|\right) / c_{0}\right)} \\
& \times \frac{R_{T}\left(\omega_{0}, \boldsymbol{z}, \boldsymbol{z}^{\prime}, \mathbf{T}\right)}{G_{i j}\left(\mathbf{z}, \mathbf{z}^{\prime}, t, \tau^{\prime}, \tau, \mu\right)} A_{\tilde{p}}\left(\boldsymbol{z}, \boldsymbol{z}^{\prime}, t, \tau^{\prime}, \tau, \mu\right) \\
& \times A_{R_{i j}}\left(\omega_{0}, \boldsymbol{z}, \boldsymbol{z}^{\prime}, t, \tau^{\prime}, \tau, \mu\right) R_{\rho}(\boldsymbol{z}) \delta\left(\boldsymbol{z}-\boldsymbol{z}^{\prime}\right) d \boldsymbol{z} d \boldsymbol{z}^{\prime} \phi(t) d t
\end{aligned}
$$

where $A_{\tilde{p}}$ is the product of the complex envelope of the transmitted waveform

$$
\begin{aligned}
A_{\tilde{p}} & =\tilde{p}\left(t+\tau^{\prime}-\left(\left|\gamma_{i}\left(t+\tau^{\prime}\right)-\mathbf{z}\right|+|\mathbf{T}-\mathbf{z}|\right) / c_{0}\right) \\
& \times \tilde{p}^{*}\left(\mu t+\tau-\left(\left|\gamma_{j}(\mu t+\tau)-\mathbf{z}^{\prime}\right|+\left|\mathbf{T}-\mathbf{z}^{\prime}\right|\right) / c_{0}\right)
\end{aligned}
$$

$A_{R_{i j}}$ is the product of the receiver antenna beam patterns

$$
\begin{gathered}
A_{R_{i j}}\left(\omega_{0}, \boldsymbol{z}, \boldsymbol{z}^{\prime}, t, \tau^{\prime}, \tau, \mu\right)=J_{\mathrm{rc}}\left(\omega_{0}, \mathbf{z}-\widehat{\gamma_{i}\left(t+\tau^{\prime}\right)}, \gamma_{i}\left(t+\tau^{\prime}\right)\right) \\
\left.\times J_{\mathrm{rc}}^{*}\left(\omega_{0}, \mathbf{z}^{\prime}-\widehat{\gamma_{j}(\mu t}+\tau\right), \gamma_{j}(\mu t+\tau)\right)
\end{gathered}
$$

and $G_{i j}$ is the product of the geometric spreading factors

$$
\begin{aligned}
G_{i j}\left(\mathbf{z}, \mathbf{z}^{\prime}, t, \tau^{\prime}, \tau, \mu\right) & =|\mathbf{T}-\mathbf{z}|\left|\mathbf{T}-\mathbf{z}^{\prime}\right| \\
\times & \times \gamma_{i}\left(t+\tau^{\prime}\right)-\mathbf{z}|| \gamma_{j}(\mu t+\tau)-\mathbf{z}^{\prime} \mid .
\end{aligned}
$$

Note that, for noncooperative sources of opportunity, $\mathbf{T}$ and thus $|\mathbf{T}-\mathbf{z}|\left|\mathbf{T}-\mathbf{z}^{\prime}\right|$ are unknown.

Now, using the Taylor series expansion at $t=0$ for $\gamma_{i}\left(t+\tau^{\prime}\right)$ and $\gamma_{j}(\mu t+\tau)$, hence approximating $\left|\gamma_{i}\left(t+\tau^{\prime}\right)-\mathbf{z}\right|$ and $\mid \gamma_{j}(\mu t+$ $\tau)-\mathbf{z}^{\prime} \mid$ in the case that $\left.\mid \gamma_{i} \dot{(} \tau^{\prime}\right) t|\ll| \gamma_{i}\left(\tau^{\prime}\right)-\mathbf{z} \mid$ and $\left.\mid \gamma_{j} \dot{(} \tau\right) \mu t \mid \ll$ $\left|\gamma_{j}(\tau)-\mathbf{z}\right|$, respectively, after the $\boldsymbol{z}^{\prime}$ integration, (12) becomes

$$
\begin{aligned}
E & {\left[c_{i j}\left(\tau^{\prime}, \tau, \mu\right)\right] } \\
& \approx \mathcal{F}_{i j}\left[R_{\rho}\right](\tau, \mu) \\
& =\int e^{-i \varphi_{i j}\left(t, \boldsymbol{z}, \tau^{\prime}, \tau, \mu\right)} A_{i j}\left(\boldsymbol{z}, t, \tau^{\prime}, \tau, \mu\right) R_{\rho}(\boldsymbol{z}) d \boldsymbol{z} d t
\end{aligned}
$$

where

$$
\begin{array}{r}
\varphi_{i j}\left(t, \boldsymbol{z}, \tau^{\prime}, \tau, \mu\right)=\omega_{0} t\left[1-\left(\boldsymbol{\gamma}_{j} \widehat{(\tau)}-\mathbf{z}\right) \cdot \dot{\gamma}_{j}(\tau) / c_{0}\right] \\
\times\left[\mu-S_{i j}\left(\tau^{\prime}, \tau, \mathbf{z}\right)\right]
\end{array}
$$

with

$$
\begin{aligned}
& S_{i j}\left(\tau^{\prime}, \tau, \mathbf{z}\right) \\
&= \frac{1-\left(\boldsymbol{\gamma}_{i} \widehat{\left(\tau^{\prime}\right)}-\mathbf{z}\right) \cdot \dot{\gamma}_{i}\left(\tau^{\prime}\right) / c_{0}}{1-\left(\boldsymbol{\gamma}_{j}(\tau)-\mathbf{z}\right) \cdot \dot{\gamma}_{j}(\tau) / c_{0}} \\
& A_{i j}\left(\boldsymbol{z}, t, \tau^{\prime}, \tau, \mu\right) \\
&=\frac{\tilde{R}_{T}\left(\omega_{0}, \boldsymbol{z}\right) \omega_{0}^{4} \phi(t)}{(4 \pi)^{4} G_{i j}\left(\mathbf{z}, \mathbf{z}, t, \tau^{\prime}, \tau, \mu\right)} \\
& \quad \times A_{\tilde{p}}\left(\boldsymbol{z}, \boldsymbol{z}, t, \tau^{\prime}, \tau, \mu\right) A_{R_{i j}}\left(\omega_{0}, \boldsymbol{z}, \boldsymbol{z}, t, \tau^{\prime}, \tau, \mu\right) \\
& \quad \times e^{i \omega_{0}\left(\tau^{\prime}-\tau-\left(\left|\boldsymbol{\gamma}_{i}\left(\tau^{\prime}\right)-\mathbf{z}\right|-\left|\boldsymbol{\gamma}_{j}(\tau)-\mathbf{z}\right|\right) / c_{0}\right)}
\end{aligned}
$$

with $\tilde{R}_{T}\left(\omega_{0}, \boldsymbol{z}\right)=R_{T}\left(\omega_{0}, \boldsymbol{z}, \boldsymbol{z}, \mathbf{T}\right)$. We refer to $S_{i j}\left(\tau^{\prime}, \tau, \mathbf{z}\right)$ as the Doppler-hitchhiker scale factor.

We refer to $\mathcal{F}_{i j}$ defined in (16) as the DSAH or Dopplerhitchhiker forward modeling operator and also to $\varphi_{i j}$ and $A_{i j}$ as the phase and amplitude terms of the linear operator $\mathcal{F}_{i j}$. Note that the scaled-and-translated correlation of the received signal removes all transmitter-related terms from the phase of the operator $\mathcal{F}_{i j}$.

For cooperative sources of opportunity where the transmitter locations and antenna beam are assumed to be known, we treat $J_{\text {tr }}$ deterministically. $\tilde{R}_{T}\left(\omega_{0}, z\right)$ in (19) is replaced with $J_{\operatorname{tr}}\left(\omega_{0}, \widehat{\mathbf{z}-\mathbf{T}}, \mathbf{T}\right) J_{\operatorname{tr}}^{*}\left(\omega_{0}, \widehat{\mathbf{z}-\mathbf{T}}, \mathbf{T}\right)$.

\section{High-Frequency Analysis of the Doppler-Hitchhiker Forward Model}

We assume that, for some $m_{A}, A_{i j}$ satisfies the inequality

$$
\begin{array}{r}
\sup _{(t, \mu, \tau, \boldsymbol{z}) \in \mathcal{U}}\left|\partial_{t}^{\alpha_{t}} \partial_{\mu}^{\alpha_{\mu}} \partial_{\tau}^{\beta} \partial_{z_{1}}^{\epsilon_{1}} \partial_{z_{2}}^{\epsilon_{2}} A_{i j}\left(\boldsymbol{z}, t, \tau^{\prime}, \tau, \mu\right)\right| \\
\leq C_{A}\left(1+t^{2}\right)^{\left(m_{A}-\left|\alpha_{t}\right|\right) / 2}
\end{array}
$$

where $\mathcal{U}$ is any compact subset of $\mathbb{R} \times \mathbb{R}^{+} \times \mathbb{R} \times \mathbb{R}^{2}$ and the constant $C_{A}$ depends on $\mathcal{U}, \alpha_{t, \mu}, \beta$, and $\epsilon_{1,2}$. This assumption is needed to make various stationary phase calculations hold. 


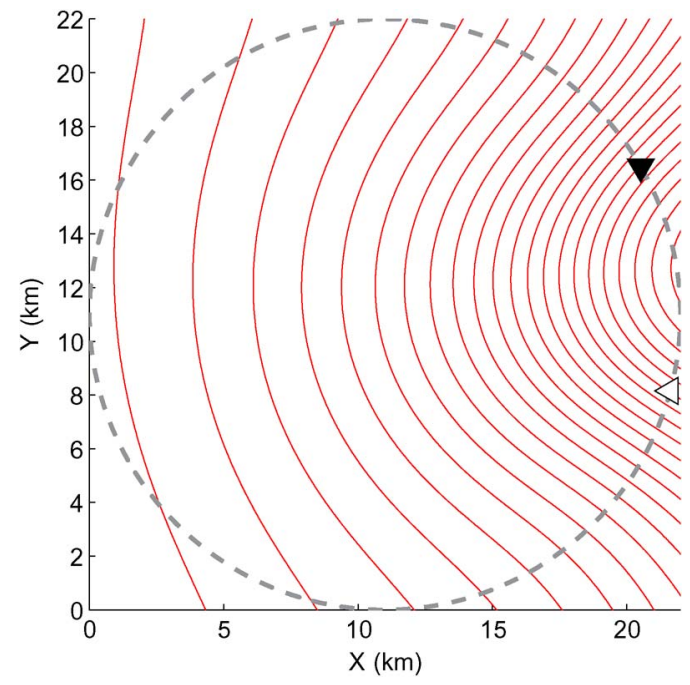

Fig. 2. Iso-Doppler contours $F_{i j}\left(\tau^{\prime}, \tau, \mu\right)$ for the Doppler-hitchhiker scale factor $S_{12}(26.1799 \mathrm{~s},-13.09 \mathrm{~s}, \mathbf{z})$. Two receivers are traversing a circular flight trajectory (dashed line) at the speed of $220 \mathrm{~m} / \mathrm{s}$ over a flat topography. $\gamma_{1}(s)=\gamma_{C}(s)$ and $\gamma_{2}(s)=\gamma_{C}(s-\pi / 4)$, respectively, where white and black triangles denote the positions of the two receivers at $s=\pi / 6$, respectively. (See (18) and (67) for explicit formulas of $S_{12}(26.1799 \mathrm{~s},-13.09 \mathrm{~s}, \mathbf{z})$ and $\gamma_{C}(s)$, respectively.)

In practice, (20) is satisfied for transmitters and receivers sufficiently far away from the illuminated region.

Under the assumption (20), (16) defines $\mathcal{F}$ as a Fourier integral operator whose leading-order contributions come from those points lying in the intersection of the illuminated surface $(\boldsymbol{z}, \boldsymbol{\psi}(\boldsymbol{z}))$ and points that have the same Doppler-hitchhiker scale factor, i.e., $\left\{\mathbf{z} \in \mathbb{R}^{3}: S_{i j}\left(\tau^{\prime}, \tau, \mathbf{z}\right)=\mu\right\}$. We denote the curves formed by this intersection by

$$
F_{i j}\left(\tau^{\prime}, \tau, \mu\right)=\left\{\boldsymbol{z}: S_{i j}\left(\tau^{\prime}, \tau, \mathbf{z}=(\boldsymbol{z}, \psi(\boldsymbol{z}))\right)=\mu\right\} .
$$

When the speed of the receivers is much slower than the speed of light $c_{0}, S_{i j}$ can be approximated as follows:

$$
\begin{aligned}
& S_{i j}\left(\tau^{\prime}, \tau, \mathbf{z}\right) \\
& \quad=1+\frac{\left(\gamma_{j} \widehat{(\tau)-} \mathbf{z}\right) \cdot \dot{\gamma}_{j}(\tau) / c_{0}-\left(\boldsymbol{\gamma}_{i} \widehat{\left(\tau^{\prime}\right)}-\mathbf{z}\right) \cdot \dot{\gamma}_{i}\left(\tau^{\prime}\right) / c_{0}}{1-\left(\gamma_{j}(\tau)-\mathbf{z}\right) \cdot \dot{\gamma}_{j}(\tau) / c_{0}} \\
& \quad \approx 1+\left[\left(\boldsymbol{\gamma}_{j} \widehat{(\tau)-} \mathbf{z}\right) \cdot \dot{\gamma}_{j}(\tau)-\left(\gamma_{i} \widehat{\left(\tau^{\prime}\right)-} \mathbf{z}\right) \cdot \dot{\gamma}_{i}\left(\tau^{\prime}\right)\right] / c_{0} .
\end{aligned}
$$

Substituting (22) into

$$
S_{i j}\left(\tau^{\prime}, \tau, \mathbf{z}\right)=\mu
$$

multiplying both sides of (23) by $\omega_{0}$, and rearranging the terms, we have

$\frac{\omega_{0}}{c_{0}}\left[\left(\gamma_{i} \widehat{\left(\tau^{\prime}\right)}-\mathbf{z}\right) \cdot \dot{\gamma}_{i}\left(\tau^{\prime}\right)-\left(\gamma_{j} \widehat{(\tau)}-\mathbf{z}\right) \cdot \dot{\gamma}_{j}(\tau)\right]=(1-\mu) \omega_{0}$

where the left-hand side of (24) is the hitchhiker Doppler defined in [19] for a fixed frequency. In this regard, we refer to $F_{i j}\left(\tau^{\prime}, \tau, \mu\right)$ as the DSAH iso-Doppler contour. Fig. 2 shows the DSAH iso-Doppler contours for two receivers traversing a circular trajectory over a flat topography.
The high-frequency analysis of the forward model shows that the windowed scaled-and-translated correlation of the received signals is the projection of the scene radiance onto the hitchhiker iso-Doppler contours.

\section{IMAGE FORMATION}

Our objective is to form an image of the scene radiance $R_{\rho}(\boldsymbol{z})$ using $E\left[c_{i j}\left(\tau^{\prime}, \tau, \mu\right)\right], i, j=1, \ldots, N$, for a range of $\tau^{\prime}$, $\tau$, and $\mu$ values based on the forward model (16).

Since $\mathcal{F}_{i j}$ is a Fourier integral operator, we form an image of the scene radiance by a suitable filtered backprojection of $E\left[c_{i j}\left(\tau^{\prime}, \tau, \mu\right)\right]$ onto $F_{i j}\left(\tau^{\prime}, \tau, \mu\right), i, j=1, \ldots, N$ [25].

\section{A. Filtered-Backprojection Operator}

We form an image of the scene radiance by the superposition of the filtered and backprojected data, $E\left[c_{i j}(\tau, \mu)\right]$, as follows:

$$
\tilde{R}_{\rho}(\boldsymbol{z})=\sum_{i j} \int \mathcal{K}_{i j}\left[E\left[c_{i j}\right]\right]\left(\boldsymbol{z}, \tau^{\prime}\right) d \tau^{\prime}
$$

where

$$
\begin{aligned}
\mathcal{K}_{i j}\left[E\left[c_{i j}\right]\right]\left(\boldsymbol{z}, \tau^{\prime}\right)=\int e^{i \varphi_{i j}\left(t, \boldsymbol{z}, \tau^{\prime}, \tau, \mu\right)} \\
\times Q_{i j}\left(\boldsymbol{z}, t, \tau^{\prime}, \tau\right) E\left[c_{i j}\left(\tau^{\prime}, \tau, \mu\right)\right] d t d \tau d \mu .
\end{aligned}
$$

We refer to $\mathcal{K}_{i j}$ as the filtered-backprojection operator with respect to the $i$ th and $j$ th receivers, with filter $Q_{i j}$ to be determined hereinafter. We will show that the filtered-backprojection operator reconstructs the visible edges of the scene at the correct location and correct orientation irrespective of the choice of the filter $Q_{i j}$.

We assume that, for some $m_{Q}, Q_{i j}$ satisfies the inequality

$$
\begin{aligned}
\sup _{\left(t, \tau^{\prime}, \tau, \boldsymbol{z}\right) \in \mathcal{U}}\left|\partial_{t}^{\alpha_{t}} \partial_{\tau^{\prime}}^{\beta_{1}} \partial_{\tau}^{\beta_{2}} \partial_{z_{1}}^{\epsilon_{1}} \partial_{z_{2}}^{\epsilon_{2}} Q_{i j}\left(\boldsymbol{z}, t, \tau^{\prime}, \tau\right)\right| \\
\leq C_{Q}\left(1+t^{2}\right)^{\left(m_{Q}-\left|\alpha_{t}\right|\right) / 2}
\end{aligned}
$$

where $\mathcal{U}$ is any compact subset of $\mathbb{R} \times \mathbb{R} \times \mathbb{R} \times \mathbb{R}^{2}$ and the constant $C_{Q}$ depends on $\mathcal{U}, \alpha_{t}, \beta_{1,2}$, and $\epsilon_{1,2}$. Assumption (27) makes $\mathcal{K}_{i j}$ a Fourier integral operator.

\section{B. PSF}

We rewrite $\tilde{R}_{\rho}$ as

$$
\tilde{R}_{\rho}(\boldsymbol{z})=\sum_{i j} \mathcal{K}_{i j} \mathcal{F}_{i j}\left[R_{\rho}\right](\boldsymbol{z})=\int L\left(\boldsymbol{z}, \boldsymbol{z}^{\prime}\right) R_{\rho}\left(\boldsymbol{z}^{\prime}\right) d \boldsymbol{z}^{\prime}
$$

where $L\left(\boldsymbol{z}, \boldsymbol{z}^{\prime}\right)$ is the PSF of the imaging operator given by

$$
\begin{aligned}
L\left(\boldsymbol{z}, \boldsymbol{z}^{\prime}\right)= & \sum_{i j} \int L_{i j}\left(\boldsymbol{z}, \boldsymbol{z}^{\prime}, \tau^{\prime}\right) d \tau^{\prime} \\
L_{i j}\left(\boldsymbol{z}, \boldsymbol{z}^{\prime}, \tau^{\prime}\right)= & \int e^{i\left[\varphi_{i j}\left(t, \boldsymbol{z}, \tau^{\prime}, \tau, \mu\right)-\varphi_{i j}\left(t^{\prime}, \boldsymbol{z}^{\prime}, \tau^{\prime}, \tau, \mu\right)\right]} \\
& \times Q_{i j}\left(\boldsymbol{z}, t, \tau^{\prime}, \tau\right) A_{i j}\left(\boldsymbol{z}^{\prime}, t^{\prime}, \tau^{\prime}, \tau, \mu\right) d t^{\prime} d t d \tau d \mu .
\end{aligned}
$$


Note that $L_{i j}\left(\boldsymbol{z}, \boldsymbol{z}^{\prime}, \tau^{\prime}\right)$ can be viewed as the PSF of the partial imaging operator $\mathcal{K}_{i j}$.

We next define

$$
\Phi_{K}:=\varphi_{i j}\left(t, \boldsymbol{z}, \tau^{\prime}, \tau, \mu\right)-\varphi_{i j}\left(t^{\prime}, \boldsymbol{z}^{\prime}, \tau^{\prime}, \tau, \mu\right)
$$

as the phase of $\mathcal{K}_{i j} \mathcal{F}_{i j}$ and use the stationary phase theorem [26]-[29] to approximate the $t^{\prime}$ and $\mu$ integrations.

The stationary points of the phase satisfy $\partial_{t^{\prime}, \mu} \Phi_{K}=0$, implying that

$$
\begin{aligned}
& \mu=S_{i j}\left(\tau^{\prime}, \tau, \mathbf{z}^{\prime}\right) \\
& t^{\prime}=t \frac{1-\left(\gamma_{j}(\tau)-\mathbf{z}\right) \cdot \dot{\gamma}_{j}(\tau) / c_{0}}{1-\left(\gamma_{j}(\tau)-\mathbf{z}^{\prime}\right) \cdot \dot{\gamma}_{j}(\tau) / c_{0}} .
\end{aligned}
$$

Substituting (32) and (33) back into (30), we obtain

$$
\begin{aligned}
& L_{i j}\left(\boldsymbol{z}, \boldsymbol{z}^{\prime}, \tau^{\prime}\right) \\
& \quad \approx \int e^{i \omega_{0} t\left[1-\left(\widehat{\left.\gamma_{j}(\tau)-\mathbf{z}\right)} \cdot \dot{\gamma}_{j}(\tau) / c_{0}\right]\left[S_{i j}\left(\tau^{\prime}, \tau, \boldsymbol{z}^{\prime}\right)-S_{i j}\left(\tau^{\prime}, \tau, \boldsymbol{z}\right)\right]\right.} \\
& \quad \times Q_{i j}\left(\boldsymbol{z}, t, \tau^{\prime}, \tau\right) A_{i j}\left(\boldsymbol{z}^{\prime}, t, \tau^{\prime}, \tau, S_{i j}\left(\tau^{\prime}, \tau, \boldsymbol{z}^{\prime}\right)\right) d t d \tau .
\end{aligned}
$$

To simplify our notation, for the rest of our paper, we write

$$
A_{i j}\left(\boldsymbol{z}, t, \tau^{\prime}, \tau\right)=A_{i j}\left(\boldsymbol{z}, t, \tau^{\prime}, \tau, S_{i j}\left(\tau^{\prime}, \tau, \boldsymbol{z}\right)\right) .
$$

Applying the method of stationary phase to the $t$ and $\tau$ integrals, we see that the main contribution to $L_{i j}\left(\boldsymbol{z}, \boldsymbol{z}^{\prime}, \tau^{\prime}\right)$ comes from those critical points of its phase that satisfy the conditions

$$
\begin{gathered}
\partial_{t}\left(\omega_{0} t\left[1-\left(\boldsymbol{\gamma}_{j} \widehat{(\tau)-} \mathbf{z}\right) \cdot \dot{\gamma}_{j}(\tau) / c_{0}\right]\right. \\
\left.\left[S_{i j}\left(\tau^{\prime}, \tau, \boldsymbol{z}^{\prime}\right)-S_{i j}\left(\tau^{\prime}, \tau, \boldsymbol{z}\right)\right]\right)=0 \\
\Rightarrow S_{i j}\left(\tau^{\prime}, \tau, \boldsymbol{z}^{\prime}\right)=S_{i j}\left(\tau^{\prime}, \tau, \boldsymbol{z}\right) \\
\partial_{\tau}\left(\omega_{0} t\left[1-\left(\boldsymbol{\gamma}_{j}^{(\tau)}-\mathbf{z}\right) \cdot \dot{\gamma}_{j}(\tau) / c_{0}\right]\right. \\
\left.\left[S_{i j}\left(\tau^{\prime}, \tau, \boldsymbol{z}^{\prime}\right)-S_{i j}\left(\tau^{\prime}, \tau, \boldsymbol{z}\right)\right]\right)=0 \\
\Rightarrow \frac{a_{j}^{\sum_{j}}(\tau, \mathbf{z})}{1-\left(\boldsymbol{\gamma}_{j}(\tau)-\mathbf{z}\right) \cdot \dot{\gamma}_{j}(\tau) / c_{0}} \\
=\frac{a_{j}^{\sum}\left(\tau, \mathbf{z}^{\prime}\right)}{1-\left(\gamma_{j} \widehat{(\tau)}-\mathbf{z}^{\prime}\right) \cdot \dot{\gamma}_{j}(\tau) / c_{0}}
\end{gathered}
$$

where

$$
\begin{aligned}
& a_{j}^{\sum_{(\tau, \mathbf{z})}}=\frac{1}{\left|\gamma_{j}(\tau)-\mathbf{z}\right|}\left|\dot{\gamma}_{j, \perp}(\tau, \mathbf{z})\right|^{2}+\left(\gamma_{j} \widehat{(\tau)}-\mathbf{z}\right) \cdot \ddot{\gamma}_{j}(\tau) \\
& \dot{\gamma}_{j, \perp}(\tau, \mathbf{z})=\dot{\gamma}_{j}(\tau)-\left(\widehat{\gamma_{j}(\tau)-} \mathbf{z}\right)\left(\left(\gamma_{j} \widehat{(\tau)}-\mathbf{z}\right) \cdot \dot{\gamma}_{j}(\tau)\right)
\end{aligned}
$$

Note that $\dot{\gamma}_{j, \perp}(\tau, \mathbf{z})$ is the projection of the receiver velocity $\dot{\gamma}_{j}(\tau)$ onto the plane whose normal direction is along

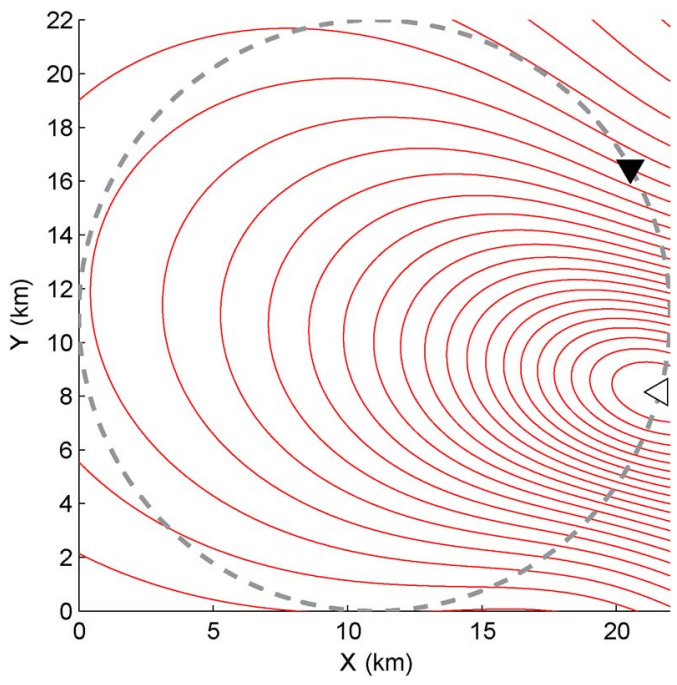

Fig. 3. Iso-Doppler-rate contours $\dot{F}_{j}(\tau, C)$ for the DSAH Doppler rate $f_{1}(-13.09 \mathrm{~s}, \mathbf{z})$. Two receivers are traversing a circular flight trajectory (dashed line) at the speed of $220 \mathrm{~m} / \mathrm{s}$ over a flat topography. $\gamma_{1}(s)=\gamma_{C}(s)$ and $\gamma_{2}(s)=\gamma_{C}(s-\pi / 4)$, respectively, where white and black triangles denote the positions of the two receivers at $s=\pi / 6$, respectively. (See (40) and (67) for explicit formulas of $f_{2}(-13.09 \mathrm{~s}, \mathbf{z})$ and $\gamma_{C}(s)$, respectively.)

$\gamma_{j} \widehat{(\tau)}-\mathbf{z}$ and $a_{j} \sum_{(\tau, \mathbf{z})}$ is the total relative radial acceleration of the $j$ th receiver in the direction of $\gamma_{j} \widehat{(\tau)}-\mathrm{z}$. For the derivation of (37), see Appendix A.

We define

$$
f_{j}(\tau, \mathbf{z}):=\frac{a_{j}^{\sum_{j}}(\tau, \mathbf{z})}{1-\left(\gamma_{j}(\tau)-\mathbf{z}\right) \cdot \dot{\gamma}_{j}(\tau) / c_{0}} .
$$

We refer to $f_{j}(\tau, \mathbf{z})$ as the DSAH Doppler rate of the $j$ th receiver. We refer to the locus of points formed by the intersection of the surface topography and $\left\{\mathbf{z} \in \mathbb{R}^{3}: f_{j}(\tau, \mathbf{z})=C\right\}$, for some constant $C$, as the DSAH iso-Doppler-rate contour and denote it by

$$
\dot{F}_{j}(\tau, C)=\left\{\boldsymbol{z}: f_{j}(\tau, \boldsymbol{z})=C\right\} .
$$

Fig. 3 shows the DSAH iso-Doppler rate contours with fixed $\tau$ for a circular flight trajectory and flat topography. The critical points $\boldsymbol{z}$ of the phase of $L_{i j}\left(\boldsymbol{z}, \boldsymbol{z}^{\prime}, \tau^{\prime}\right)$ are those points lying on the intersection of the DSAH iso-Doppler curves $F_{i j}\left(\tau^{\prime}, \tau, \mu\right)$ and DSAH iso-Doppler-rate curves $\dot{F}_{j}(\tau, C)$. We assume that the flight trajectories of the receivers are smooth and that the receiver antenna beam patterns are focused on a region of interest where each pair of iso-Doppler $F_{i j}\left(\tau^{\prime}, \tau, \mu\right)$ and iso-Doppler-rate $\dot{F}_{j}(\tau, C)$ contours intersects at a single point within the region of interest. In other words, we assume that the only critical point within the region of interest is $\boldsymbol{z}=\boldsymbol{z}^{\prime}$.

\section{Determination of the Filter}

The filter $Q_{i j}$ can be determined with respect to various criteria [30]. Ideally, $Q_{i j}$ is chosen such that the leading-order contributions of the PSF of $\mathcal{K}_{i j}$ are the Dirac-delta function, i.e., $L_{i j}\left(\boldsymbol{z}, \boldsymbol{z}^{\prime}, \tau^{\prime}\right) \approx \delta\left(\boldsymbol{z}-\boldsymbol{z}^{\prime}\right)$. This choice of $Q_{i j}$ ensures that 
$\mathcal{K}_{i j}$ reconstructs the visible edges of the scene not only at the right location and orientation but also with the correct strength [23], [30]-[32].

To determine the filter, we linearize $S_{i j}\left(\tau^{\prime}, \tau, \boldsymbol{z}^{\prime}\right)$ around $\boldsymbol{z}^{\prime}=\boldsymbol{z}$ and approximate

$$
\begin{aligned}
S_{i j}\left(\tau^{\prime}, \tau, \boldsymbol{z}^{\prime}\right)-S_{i j}\left(\tau^{\prime}, \tau, \boldsymbol{z}\right) & \approx \nabla_{\boldsymbol{z}} S_{i j}\left(\tau^{\prime}, \tau, \boldsymbol{z}\right) \cdot\left(\boldsymbol{z}^{\prime}-\boldsymbol{z}\right) \\
A_{i j}\left(\boldsymbol{z}^{\prime}, t, \tau^{\prime}, \tau\right) & \approx A_{i j}\left(\boldsymbol{z}, t, \tau^{\prime}, \tau\right)
\end{aligned}
$$

to write

$$
\begin{aligned}
L_{i j}\left(\boldsymbol{z}, \boldsymbol{z}^{\prime}, \tau^{\prime}\right)=\int & e^{i t \boldsymbol{\Xi}_{i j}\left(\tau^{\prime}, \tau, \boldsymbol{z}\right) \cdot\left(\boldsymbol{z}^{\prime}-\boldsymbol{z}\right)} \\
& \times Q_{i j}\left(\boldsymbol{z}, t, \tau^{\prime}, \tau\right) A_{i j}\left(\boldsymbol{z}, t, \tau^{\prime}, \tau\right) d t d \tau
\end{aligned}
$$

where

$$
\boldsymbol{\Xi}_{i j}\left(\tau^{\prime}, \tau, \boldsymbol{z}\right)=\omega_{0}\left[1-\left(\widehat{\gamma_{j}(\tau)-\mathbf{z}}\right) \cdot \dot{\gamma}_{j}(\tau) / c_{0}\right] \nabla_{\boldsymbol{z}} S_{i j}\left(\tau^{\prime}, \tau, \boldsymbol{z}\right) \text {. }
$$

In (44), for each $\tau^{\prime}$ and $z$, we make the following change of variables:

$$
(t, \tau) \rightarrow \boldsymbol{\xi}_{i j}=t \boldsymbol{\Xi}_{i j}\left(\tau^{\prime}, \tau, \boldsymbol{z}\right)
$$

and obtain

$$
\begin{aligned}
& L_{i j}\left(\boldsymbol{z}, \boldsymbol{z}^{\prime}, \tau^{\prime}\right)=\int_{\Omega_{i j, \tau^{\prime}, \boldsymbol{z}}} e^{i \boldsymbol{\xi}_{i j} \cdot\left(\boldsymbol{z}^{\prime}-\boldsymbol{z}\right)} \\
& \quad \times Q_{i j}\left(\boldsymbol{z}, \tau^{\prime}, \boldsymbol{\xi}_{i j}\right) A_{i j}\left(\boldsymbol{z}, \tau^{\prime}, \boldsymbol{\xi}_{i j}\right) \eta\left(\boldsymbol{z}, \tau^{\prime}, \boldsymbol{\xi}_{i j}\right) d \boldsymbol{\xi}_{i j},
\end{aligned}
$$

where

$$
\begin{aligned}
& Q_{i j}\left(\boldsymbol{z}, \tau^{\prime}, \boldsymbol{\xi}_{i j}\right)=Q_{i j}\left(\boldsymbol{z}, t\left(\boldsymbol{\xi}_{i j}\right), \tau^{\prime}, \tau\left(\boldsymbol{\xi}_{i j}\right)\right) \\
& A_{i j}\left(\boldsymbol{z}, \tau^{\prime}, \boldsymbol{\xi}_{i j}\right)=A_{i j}\left(\boldsymbol{z}, t\left(\boldsymbol{\xi}_{i j}\right), \tau^{\prime}, \tau\left(\boldsymbol{\xi}_{i j}\right)\right)
\end{aligned}
$$

and $\eta\left(\boldsymbol{z}, \tau^{\prime}, \boldsymbol{\xi}_{i j}\right)=\left|\partial(t, \tau) / \partial \boldsymbol{\xi}_{i j}\right|$ is the determinant of the Jacobian that comes from the change variables in (46).

In (47)

$$
\begin{array}{r}
\Omega_{i j, \tau^{\prime}, \boldsymbol{z}}=\left\{\boldsymbol{\xi}_{i j}=t \boldsymbol{\Xi}_{i j}\left(\tau^{\prime}, \tau, \boldsymbol{z}\right) \mid A_{i j}\left(\boldsymbol{z}, t, \tau^{\prime}, \tau\right) \neq 0,\right. \\
\left.\left(t, \tau^{\prime}, \tau\right) \in(\mathbb{R}, \mathbb{R}, \mathbb{R})\right\} .
\end{array}
$$

We refer to $\Omega_{i j, \tau^{\prime}, \boldsymbol{z}}$ as the partial data collection manifold at $\left(\tau^{\prime}, \boldsymbol{z}\right)$ obtained by the $i$ th and $j$ th receivers for a fixed $\tau^{\prime}$ and refer to the union $\cup_{i j, \tau^{\prime}} \Omega_{i j, \tau^{\prime}, z}$ as the data collection manifold at $z$ and denote it by $\Omega_{z}$. This set determines many of the properties of the image.

Using (18) and (45), we obtain

$$
\begin{aligned}
& \boldsymbol{\Xi}_{i j}\left(\tau^{\prime}, \tau, \boldsymbol{z}\right)=\frac{\omega_{0}}{c_{0}} D \psi(\boldsymbol{z}) \\
& \cdot\left[\frac{1}{\left|\boldsymbol{\gamma}_{i}\left(\tau^{\prime}\right)-\mathbf{z}\right|} \dot{\gamma}_{i, \perp}\left(\tau^{\prime}\right)-\frac{S_{i j}\left(\tau^{\prime}, \tau, \boldsymbol{z}\right)}{\left|\boldsymbol{\gamma}_{j}(\tau)-\mathbf{z}\right|} \dot{\gamma}_{j, \perp}(\tau)\right]
\end{aligned}
$$

where

$$
D \psi(\boldsymbol{z})=\left[\begin{array}{lll}
1 & 0 & \partial \psi(\boldsymbol{z}) / \partial z_{1} \\
0 & 1 & \partial \psi(\boldsymbol{z}) / \partial z_{2}
\end{array}\right]
$$

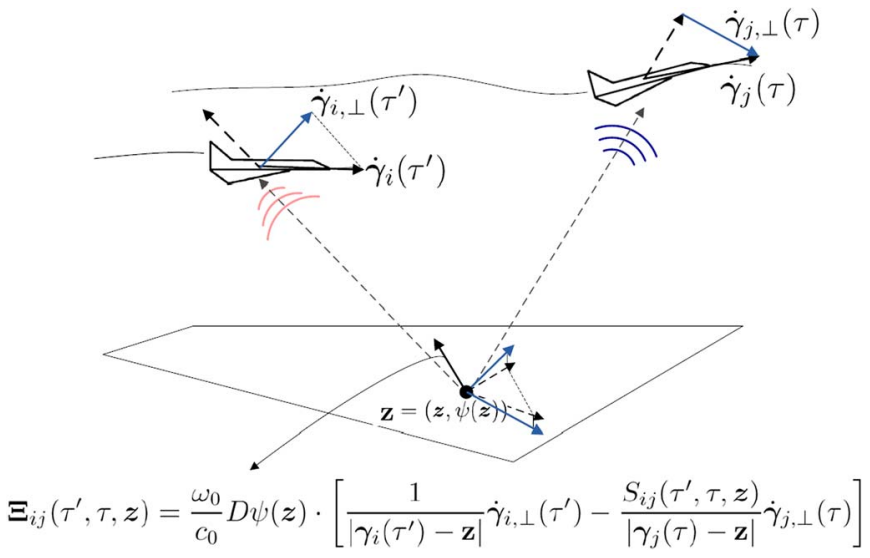

Fig. 4. Illustration of the vector $\boldsymbol{\Xi}_{i j}\left(\tau^{\prime}, \tau, \mathbf{z}\right)$ in the data collection manifold $\Omega_{i j, \mathbf{z}}$ for the flat topography, $\psi(\mathbf{z})=0 . \boldsymbol{\Xi}_{i j}\left(\tau^{\prime}, \tau, \mathbf{z}\right)$ is the projection of the difference of the scaled vectors $\dot{\gamma}_{i, \perp}\left(\tau^{\prime}\right)$ and $\dot{\gamma}_{j, \perp}(\tau)$ onto the tangent plane of the ground topography at $\mathbf{z}$. (See (51) for an explicit form of $\boldsymbol{\Xi}_{i j}\left(\tau^{\prime}, \tau, \mathbf{z}\right)$.)

and $\dot{\gamma}_{i, \perp}\left(\tau^{\prime}\right)$ and $\dot{\gamma}_{j, \perp}(\tau)$ are the projections of $\dot{\gamma}_{i}\left(\tau^{\prime}\right)$ and $\dot{\gamma}_{j}(\tau)$ onto the planes whose normal vectors are $\left(\gamma_{i} \widehat{\left(\tau^{\prime}\right)}-\mathbf{z}\right)$ and $\left(\gamma_{j} \widehat{(\tau)}-\mathbf{z}\right)$, respectively. For a detailed derivation of (51), see Appendix B.

We show an illustration of the vector $\boldsymbol{\Xi}_{i j}\left(\tau^{\prime}, \tau, \boldsymbol{z}\right)$ in Fig. 4 for two receivers flying over a flat topography.

We choose the filter as follows:

$$
Q_{i j}\left(\boldsymbol{z}, t, \tau^{\prime}, \tau\right)=\frac{A_{i j}^{*}\left(\boldsymbol{z}, t, \tau^{\prime}, \tau\right)}{\left|A_{i j}\left(\boldsymbol{z}, t, \tau^{\prime}, \tau\right)\right|^{2}} \frac{\chi_{\Omega_{i j, \tau^{\prime}, \boldsymbol{z}}}\left(\boldsymbol{z}, t, \tau^{\prime}, \tau\right)}{\eta\left(\boldsymbol{z}, \tau^{\prime}, \boldsymbol{\xi}_{i j}\right)}
$$

where $\chi_{\Omega_{i j, \tau^{\prime}, z}}$ is a smooth cutoff function equal to one in the interior of $\Omega_{i j, \tau^{\prime}, z}$ and zero in the exterior of $\Omega_{i j, \tau^{\prime}, z}$.

Note that this choice of filter makes the leading-order term of $L_{i j}\left(\boldsymbol{z}, \boldsymbol{z}^{\prime}, \tau^{\prime}\right)$ in (47) to be the Dirac-delta function.

\section{Resolution Analysis}

Substituting (53) into (47) and the result into (28), we obtain

$$
\begin{aligned}
\tilde{R}_{\rho}(\boldsymbol{z}) & =\sum_{i j} \mathcal{K}_{i j}\left[\mathcal{F}_{i j}\left[R_{\rho}\right]\right](\boldsymbol{z}) \\
& \approx \sum_{i j} \int_{\Omega_{i j, \tau^{\prime}, \boldsymbol{z}}} e^{i \boldsymbol{\xi}_{i j} \cdot\left(\boldsymbol{z}^{\prime}-\boldsymbol{z}\right)} R_{\rho}\left(\boldsymbol{z}^{\prime}\right) d \boldsymbol{z}^{\prime} d \boldsymbol{\xi}_{i j} d \tau^{\prime} .
\end{aligned}
$$

Equation (54) shows that the image $\tilde{R}_{\rho}$ is a band-limited version of $R_{\rho}$ whose bandwidth is determined by the data collection manifold $\Omega_{z}$, which describes the resolution of the reconstructed image $\tilde{R}_{\rho}$ at $z$. The larger the data collection manifold, the better the resolution of the reconstructed image is.

Microlocal analysis of (54) tells us that an edge at point $z$ is visible if the direction $\boldsymbol{n}_{\boldsymbol{z}}$ normal to the edge is contained in $\Omega_{z}$ [23], [30]-[32]. Consequently, an edge at point $\boldsymbol{z}$ with $\boldsymbol{n}_{\boldsymbol{z}}$ normal to edge is visible if there exists $i, j, \tau^{\prime}$, and $\tau$ such that $\boldsymbol{\xi}_{i j}$ is parallel to $\boldsymbol{n}_{\boldsymbol{z}}$. Furthermore, the bandwidth contribution of $\boldsymbol{\xi}_{i j}=t \boldsymbol{\Xi}_{i j}\left(\tau^{\prime}, \tau, \boldsymbol{z}\right)$ to a visible edge at $\boldsymbol{z}$ is given by $L_{\phi}\left|\boldsymbol{\Xi}_{i j}\left(\tau^{\prime}, \tau, \boldsymbol{z}\right)\right|$, where $L_{\phi}$ denotes the length of the support of $\phi(t)$. Thus, the longer the support of $\phi(t)$ becomes, the larger 


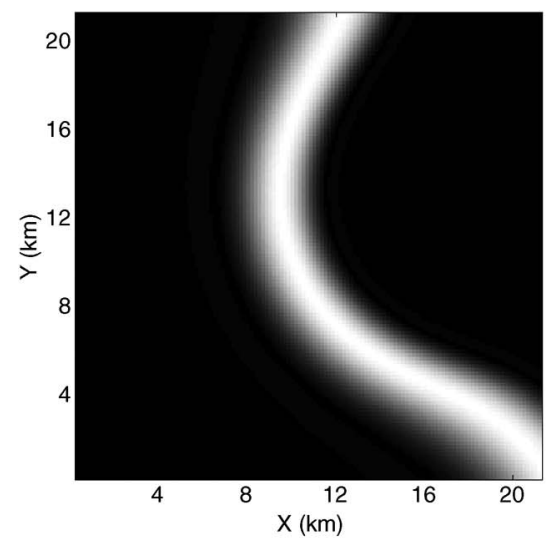

(a)

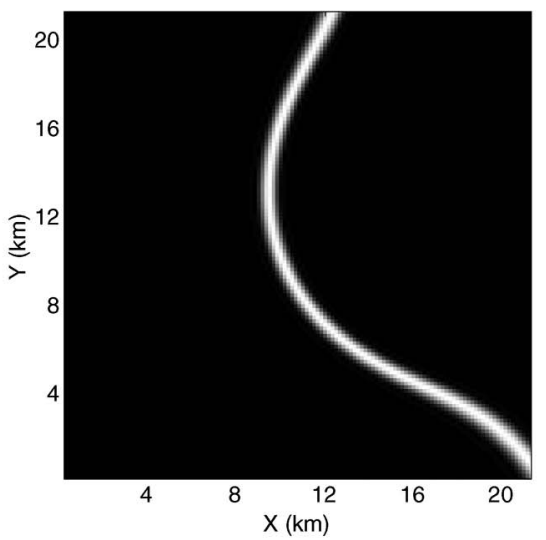

(b)

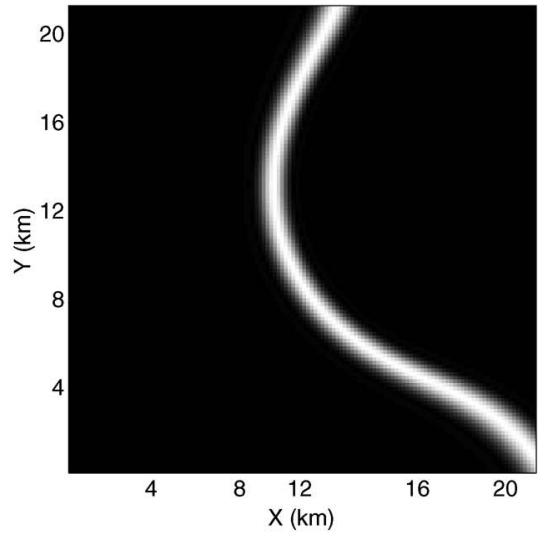

(c)

Fig. 5. DSAH iso-Doppler contours obtained by backprojecting the data for a fixed $\tau$ and $\tau^{\prime}$ with (a) $L_{\phi}=0.0853 \mathrm{~s}, f_{0}=\omega_{0} / 2 \pi=200 \mathrm{MHz}$; (b) $L_{\phi}=$ $0.6827 \mathrm{~s}, f_{0}=\omega_{0} / 2 \pi=200 \mathrm{MHz}$; and (c) $L_{\phi}=0.0853 \mathrm{~s}, f_{0}=\omega_{0} / 2 \pi=500 \mathrm{MHz}$. The data are generated using (4) for a point target located at $[9.625$,

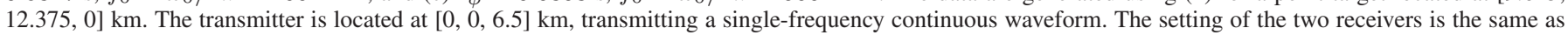
that in Fig. 2.

the magnitude of $\boldsymbol{\xi}_{i j}$ is, giving rise to sharper reconstructed edges perpendicular to $\boldsymbol{\xi}_{i j}, i, j=1, \ldots, N$. Additionally, the higher the carrier frequency $\omega_{0}$ of the transmitted signal becomes, the larger the magnitude of $\boldsymbol{\xi}_{i j}$ is, contributing to higher image resolution.

We note that the magnitude of $\boldsymbol{\xi}_{i j}$ also depends on the speed of the receivers as well as the distance of the receivers to the scatterers. The larger the magnitude of $\dot{\gamma}_{i, \perp}\left(\tau^{\prime}\right)$ and/or $\dot{\gamma}_{j, \perp}(\tau)$, the larger the magnitude of $\boldsymbol{\xi}_{i j}$ becomes. Equation (51) also tells us that certain receiver flight trajectories where

$$
\frac{1}{\left|\gamma_{i}\left(\tau^{\prime}\right)-\mathbf{z}\right|} \dot{\gamma}_{i, \perp}\left(\tau^{\prime}\right) \approx \frac{S_{i j}\left(\tau^{\prime}, \tau, \boldsymbol{z}\right)}{\left|\gamma_{j}(\tau)-\mathbf{z}\right|} \dot{\gamma}_{j, \perp}(\tau)
$$

do not provide information about the ground radiance at $\mathbf{z}$ and should be avoided.

The dependence of the image resolution on the length of the support of the windowing function and the carrier frequency of the transmitted waveform can also be understood from the perspective of the spreading of the DSAH iso-Doppler curves. As previously described, our imaging method performs the filtered-backprojection onto the DSAH iso-Doppler curves as defined by (21). The image resolution is accordingly closely related to the width of the spreading of the DSAH iso-Doppler curves, which is determined by the Doppler ambiguity of the transmitted waveform, and thus the duration of the signal to be processed, and the transmitter frequency.

We performed simulations to show the effects of the Doppler ambiguity and the frequency of the transmitted waveform on the width of the spreading of the iso-Doppler curves. As shown in Fig. 5, the spreading of the iso-Doppler curve becomes narrow with a longer windowing function or a higher transmitter frequency, which implies a better image resolution.

Note that (54) shows that, irrespective of the choice of the filter, the backprojection operator recovers the visible edges of the scene radiance at the right location for given $i$ th and $j$ th receivers and a fixed $\tau^{\prime}$. With the choice of the filter given in (53), the resulting image formation algorithm recovers the visible edges of the scene radiance not only at the correct location and orientation but also at the correct strength.

\section{Reconstruction Algorithm}

In this section, we describe the numerical implementation of our filtered-backprojection method. We implemented the inversion formulas (25), (26), and (53). Let $\boldsymbol{\Xi}_{i j}\left(\tau^{\prime}, \tau, \boldsymbol{z}\right)=$ $\left(\boldsymbol{\Xi}_{i j, 1}, \boldsymbol{\Xi}_{i j, 2}\right)$ and $\partial_{\tau} \boldsymbol{\Xi}_{i j}\left(\tau^{\prime}, \tau, \boldsymbol{z}\right)=\left(\dot{\boldsymbol{\Xi}}_{i j, 1}, \dot{\boldsymbol{\Xi}}_{i j, 2}\right)$. Then, using (46), we have

$$
\begin{aligned}
\frac{1}{\eta\left(\boldsymbol{z}, \tau^{\prime}, \boldsymbol{\xi}_{i j}\right)} & =\left|\frac{\partial \boldsymbol{\xi}_{i j}}{\partial(t, \tau)}\right| \\
& =|t|\left|\boldsymbol{\Xi}_{i j, 1} \dot{\boldsymbol{\Xi}}_{i j, 2}-\dot{\boldsymbol{\Xi}}_{i j, 1} \boldsymbol{\Xi}_{i j, 2}\right| .
\end{aligned}
$$

We refer to $|t|$ in (56) as the time-domain ramp filter, which has a similar effect on the reconstructed image as the well-known ramp filter in the tomography literature [26]. The image formed without the time-domain ramp filtering has smooth edges.

We write the filter (53) as

$$
Q_{i j}\left(\boldsymbol{z}, t, \tau^{\prime}, \tau\right)=Q_{i j, 1}\left(\boldsymbol{z}, \tau^{\prime}, \tau\right) Q_{i j, 2}\left(\boldsymbol{z}, t, \tau^{\prime}, \tau\right)|t|
$$

where

$$
\begin{aligned}
Q_{i j, 1}\left(\boldsymbol{z}, \tau^{\prime}, \tau\right) & =\left|\boldsymbol{\Xi}_{i j, 1} \dot{\boldsymbol{\Xi}}_{i j, 2}-\dot{\boldsymbol{\Xi}}_{i j, 1} \boldsymbol{\Xi}_{i j, 2}\right| \\
Q_{i j, 2}\left(\boldsymbol{z}, t, \tau^{\prime}, \tau\right) & =\chi_{\Omega_{i j, \tau^{\prime}, \boldsymbol{z}}} \frac{A_{i j}^{*}\left(\boldsymbol{z}, t, \tau^{\prime}, \tau\right)}{\left|A_{i j}\left(\boldsymbol{z}, t, \tau^{\prime}, \tau\right)\right|^{2}} .
\end{aligned}
$$

Using (25), (26), and (57), we obtain the following reconstruction formula:

$$
\begin{aligned}
& \tilde{R}_{\rho}(\boldsymbol{z})=\sum_{i j} \int e^{-i \omega_{0} t\left[1-\left(\widehat{\gamma_{j}(\tau)-z}\right) \cdot \dot{\gamma}_{j}(\tau) / c_{0}\right] S_{i j}\left(\tau^{\prime}, \tau, \boldsymbol{z}\right)}|t| \\
& \quad \times C_{i j}\left(\tau^{\prime}, \tau, t\right) Q_{i j, 2}\left(\boldsymbol{z}, t, \tau^{\prime}, \tau\right) Q_{i j, 1}\left(\boldsymbol{z}, \tau^{\prime}, \tau\right) d t d \tau d \tau^{\prime}
\end{aligned}
$$

where

$$
C_{i j}\left(\tau^{\prime}, \tau, t\right)=\int e^{i \omega_{0} t\left[1-\left(\widehat{\gamma_{j}(\tau)-z}\right) \cdot \dot{\gamma}_{j}(\tau) / c_{0}\right] \mu} E\left[c_{i j}\left(\tau^{\prime}, \tau, \mu\right)\right] d \mu .
$$

Note that, for notational simplicity, we write $C_{i j}\left(\tau^{\prime}, \tau, t\right)=$ $C_{i j}\left(\tau^{\prime}, \tau, t\left[1-\left(\gamma_{j} \widehat{(\tau)-} \mathbf{z}\right) \cdot \dot{\gamma}_{j}(\tau) / c_{0}\right]\right)$. 
Thus, for each pair of receivers, the steps of the reconstruction algorithm are as follows.

1) Compute the inverse Fourier transform of the scaled, translated, and correlated data with respect to $\mu$ according to (61), and obtain $C_{i j}\left(\tau^{\prime}, \tau, t\right)$.

2) Time-domain ramp filtering

$$
\tilde{C}_{i j}\left(\tau^{\prime}, \tau, t\right)=|t| C_{i j}\left(\tau^{\prime}, \tau, t\right) .
$$

3) Filtering with $Q_{i j, 2}$

$$
\tilde{C}_{i j, Q_{i j, 2}}\left(\boldsymbol{z}, t, \tau^{\prime}, \tau\right)=\tilde{C}_{i j}\left(\tau^{\prime}, \tau, t\right) Q_{i j, 2}\left(\boldsymbol{z}, t, \tau^{\prime}, \tau\right) .
$$

4) Backprojection step

$$
\begin{array}{r}
\tilde{R}_{\rho_{i j, 2}}\left(\boldsymbol{z}, \tau^{\prime}, \tau\right)=\int e^{-i \omega_{0} t\left[1-\left(\boldsymbol{\gamma}_{j}(\tau)-\mathbf{z}\right) \cdot \dot{\gamma}_{j}(\tau) / c_{0}\right] S_{i j}\left(\tau^{\prime}, \tau, \boldsymbol{z}\right)} \\
\times \tilde{C}_{i j, Q_{i j, 2}}\left(\boldsymbol{z}, t, \tau^{\prime}, \tau\right) d t .
\end{array}
$$

Note that $\quad \tilde{C}_{i j, Q_{i j, 2}}\left(\boldsymbol{z}, t, \tau^{\prime}, \tau\right)=\tilde{C}_{i j, Q_{i j, 2}}(\boldsymbol{z}, t[1-$ $\left.\left.\left(\gamma_{j} \widehat{(\tau)}-\mathbf{z}\right) \cdot \dot{\gamma}_{j}(\tau) / c_{0}\right], \tau^{\prime}, \tau\right)$. Equation (64) can be computed using the fast Fourier transform.

5) Partial image formation: We form the partial image $\tilde{R}_{\rho_{i j}}$ by

$$
\tilde{R}_{\rho_{i j}}(\boldsymbol{z})=\int Q_{i j, 1}\left(\boldsymbol{z}, \tau^{\prime}, \tau\right) \tilde{R}_{\rho_{i j, 2}}\left(\boldsymbol{z}, \tau^{\prime}, \tau\right) d \tau^{\prime} d \tau .
$$

Finally, we form our image $\tilde{R}_{\rho}$ by summing over all partial images

$$
\tilde{R}_{\rho}(\boldsymbol{z})=\sum_{i j} \tilde{R}_{\rho_{i j}}(\boldsymbol{z}) .
$$

Fig. 6 shows the block diagram of the imaging algorithm.

\section{NumericAl Simulations}

We conducted two sets of numerical simulations. In the first set of simulations, we numerically studied the PSF of the imaging operator under different scenarios and demonstrated the theoretical results described in Section III-C. In the second set of simulations, we demonstrated the performance of our imaging method for a multiple-point-target model.

We used the digital audio broadcasting (DAB) signals used by radio stations as the waveform of opportunity in the numerical simulations. We simulated the DAB signal based on the European standard, which uses the COded Orthogonal Frequency Division Multiplexing modulation. The main characteristics of the DAB signal are as follows: 1) The symbol has 1-ms useful duration with a guard interval of $0.246 \mathrm{~ms}$; 2) 1536 subcarriers are transmitted simultaneously per symbol; and 3) the quadrature phase shift keying coding is used for each subcarrier. The bandwidth of the DAB signal is $1.5 \mathrm{MHz}$. In the simulations, the transmitter was assumed to be operating in the VHF band at a transmission frequency of $200 \mathrm{MHz}$.

We considered a scene of size $[0,11] \times[0,11] \mathrm{km}^{2}$ with flat topography. The scene was discretized by $128 \times 128$ pixels, where $[0,0,0] \mathrm{km}$ and $[11,11,0] \mathrm{km}$ correspond to the pixels $(1,1)$ and $(128,128)$, respectively.

In all the numerical experiments, we used two airborne receivers and a single stationary transmitter operating either

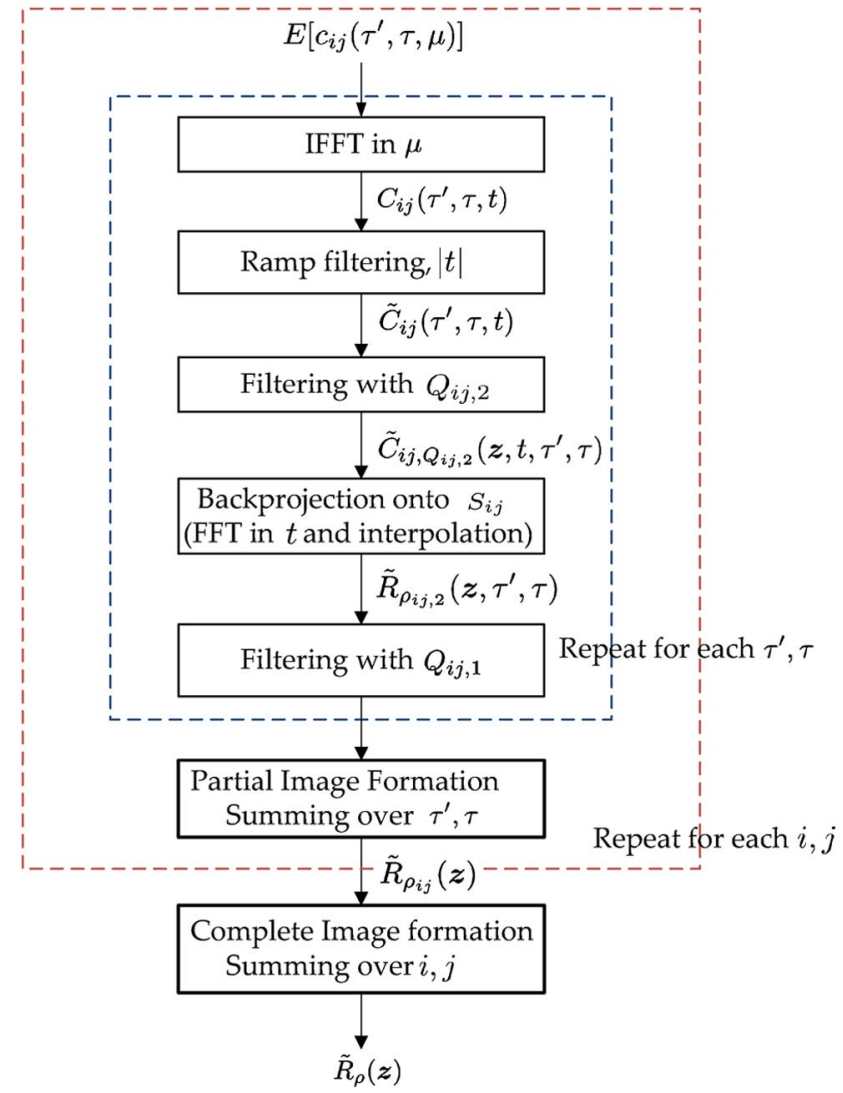

Fig. 6. Block diagram of the imaging algorithm.

cooperatively or noncooperatively. We assumed that both the receiver and transmitter antennas were isotropic. We assumed that the transmitter was located at $\mathbf{y}_{0}=(0,0,6.5) \mathrm{km}$ and the receivers were traversing the circular trajectory given by

$$
\gamma_{C}(s)=(11+11 \cos (s), 11+11 \sin (s), 6.5) \mathrm{km} .
$$

Let $\gamma_{1}(s)$ and $\gamma_{2}(s)$ denote the trajectories of the two receivers. We set $\gamma_{1}(s)=\gamma_{C}(s)$ and $\gamma_{2}(s)=\gamma_{1}(s-(\pi / 4))$. Note that the variable $s$ in $\gamma_{C}$ is equal to $(V / R) t$, where $V$ is the speed of the receiver and $R$ is the radius of the circular trajectory. We set the speed of the two receivers to $220 \mathrm{~m} / \mathrm{s}$. We chose the sampling rate of $\tau$ to be $0.8149 \mathrm{~Hz}$ so as to uniformly sample the circular trajectory with 256 points.

For all the numerical experiments, we used (4) to generate the data and chose the windowing function $\phi$ in (7) to be a Hanning function.

We performed image reconstruction for each $\tau^{\prime}$ and coherently superimposed the reconstructed images obtained over a range of $\tau^{\prime}$.

\section{A. Numerical Analysis of the PSF}

We placed a point target with unit reflectivity at [9.625, $12.375,0] \mathrm{km}$ in the scene considered. Note that this position corresponds to the $(49,81)$ th pixel in the reconstructed scene.

We performed numerical simulations to demonstrate the impact of $L_{\phi}$, the length of the support of $\phi(t) ; f_{0}=\omega_{0} / 2 \pi$, the frequency of the transmitted waveform; and the range of $\tau^{\prime}$ on the PSF of the imaging operator. We reconstructed the PSF with 


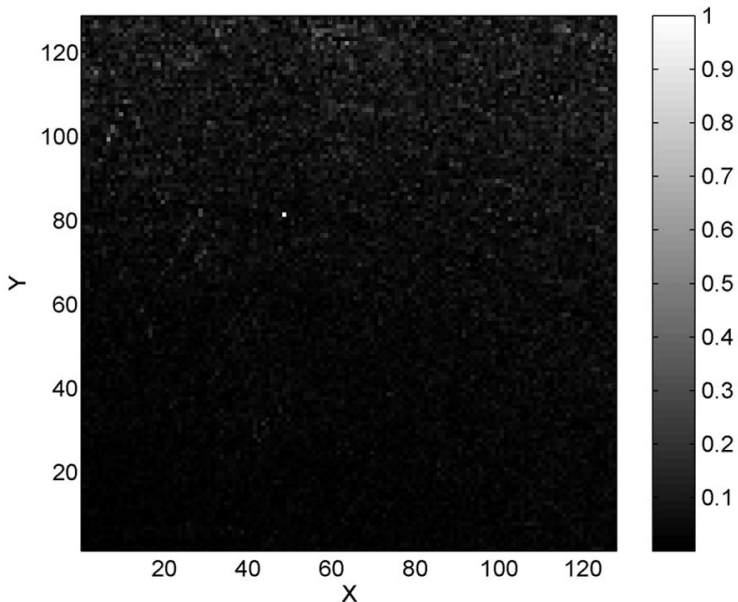

(a)

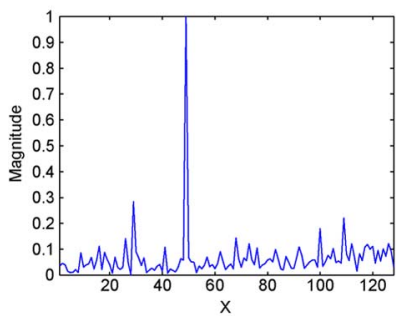

(b)

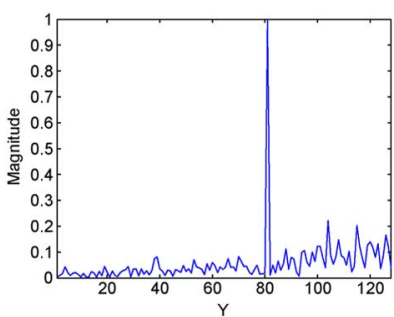

(c)
Fig. 7. (a) PSF of the DSAH imaging method at $(9.625,12.375,0) \mathrm{km}$ along with (b) its $X$ profile and (c) $Y$ profile for a cooperative DAB transmitter located at $\mathbf{y}_{0}$, transmitting a DAB waveform at $f_{0}=200 \mathrm{MHz}$, and two receivers traversing circular trajectories $\gamma_{1}(s)$ and $\gamma_{2}(s)$. The length of the support of the windowing function is $L_{\phi}=0.1707 \mathrm{~s}$.

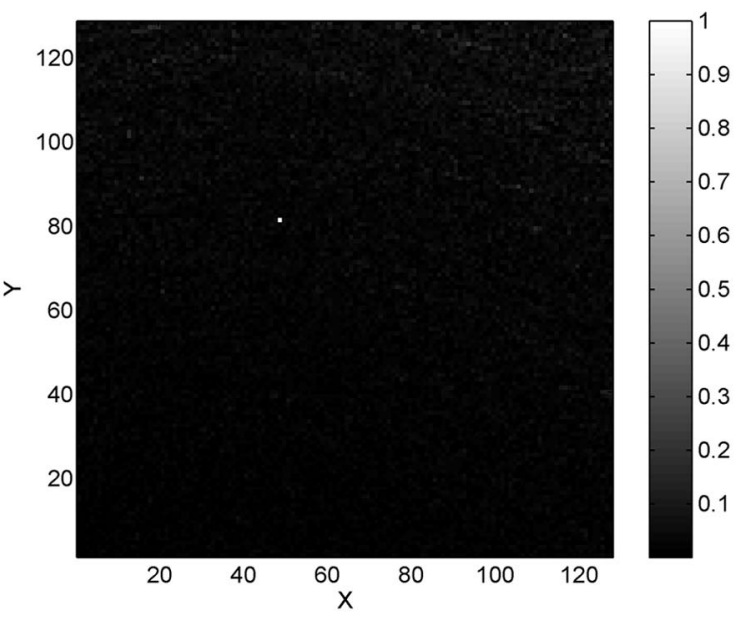

(a)

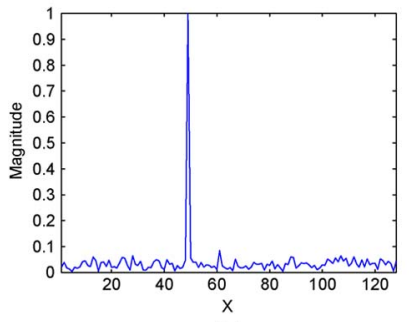

(b)

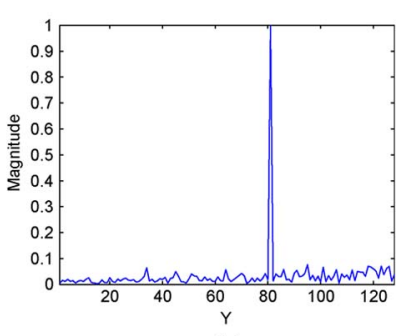

(c)
Fig. 8. (a) PSF of the DSAH imaging method at $(9.625,12.375,0) \mathrm{km}$ along with (b) its $X$ profile and (c) $Y$ profile for a cooperative DAB transmitter located at $\mathbf{y}_{0}$, transmitting a DAB waveform at $f_{0}=200 \mathrm{MHz}$, and two receivers traversing circular trajectories $\gamma_{1}(s)$ and $\gamma_{2}(s)$. The length of the support of the windowing function is $L_{\phi}=0.6827 \mathrm{~s}$.

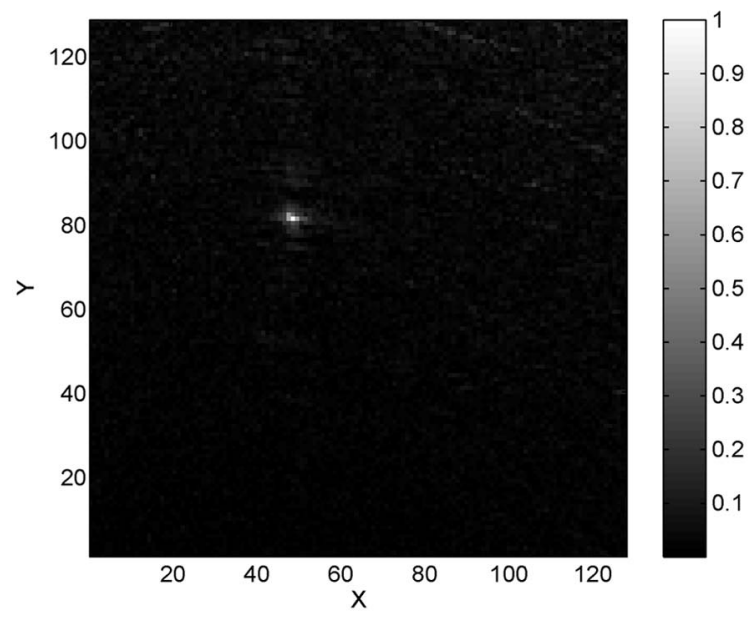

(a)

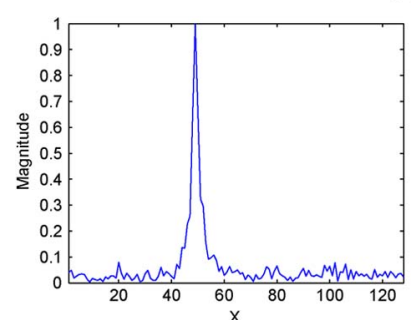

(b)

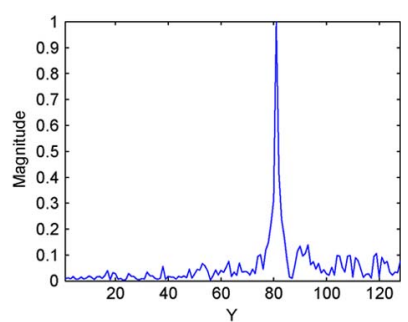

(c)
Fig. 9. (a) PSF of the DSAH imaging method at $(9.625,12.375,0) \mathrm{km}$ along with (b) its $X$ profile and (c) $Y$ profile for a cooperative DAB transmitter located at $\mathbf{y}_{0}$, transmitting a DAB waveform at $f_{0}=20 \mathrm{MHz}$, and two receivers traversing circular trajectories $\gamma_{1}(s)$ and $\gamma_{2}(s)$. The length of the support of the windowing function is $L_{\phi}=0.3413 \mathrm{~s}$.

the following variables: 1) $f_{0}=200 \mathrm{MHz}, L_{\phi}=0.1707 \mathrm{~s}$; 2) $f_{0}=200 \mathrm{MHz}, L_{\phi}=0.6827 \mathrm{~s}$; 3) $f_{0}=20 \mathrm{MHz}, L_{\phi}=0.6827 \mathrm{~s}$; and 4) $\tau^{\prime} \in[0,314.159] \mathrm{s}$ with the sampling period of $19.635 \mathrm{~s}$, $L_{\phi}=0.3413 \mathrm{~s}$, and $f_{0}=200 \mathrm{MHz}$. For the first three cases, we assumed that the transmitter was cooperative and reconstructed the PSF using $\tau^{\prime}=255.254 \mathrm{~s}$. For the fourth case, we reconstructed the PSF for both the cooperative and noncooperative cases. We used $16 \tau^{\prime}$ values uniformly spaced in [0,314.159] $\mathrm{s}$.

Figs. 7-9 show the reconstructed PSFs along with their profiles in $X$ - and $Y$-directions for the first three cases. We also tabulated the 3-dB mainlobe width and the peak-to-sidelobe ratio (PSLR) of the $X$ and $Y$ profiles in Table II, where the $3-\mathrm{dB}$ mainlobe width is used as a measure of resolution and PSLR is used as a measure of the level of the sidelobes in the reconstructed PSFs.

As can be seen in Figs. 7 and 8, the quality of the PSF improves as the length of the support of the windowing function increases from 0.1707 to $0.6827 \mathrm{~s}$ due to a larger data collection manifold. Comparing the profiles of the PSFs in both cases, we see that the level of the sidelobes of the PSF decreases as the support of the windowing function gets longer, which is also indicated by the PSLR values in the two cases in Table II. The low level of sidelobes, in turn, results in an improved visual contrast in the image, as shown in Fig. 8(a).

Note that the change in the resolution is not evident visually when the images in Figs. 7 and 8 are compared. This may be due to a relatively large pixel size of the reconstructed image and a relatively small increase in the resolution. We can see 
TABLE II

Mainlobe Width of 3 dB ANd PSLR of the ReConstructed PSFs for Cases 1, 2, ANd 3

\begin{tabular}{l|cc|cc}
\hline & \multicolumn{2}{|c|}{ X direction } & Y direction \\
Cases & 3dB Mainlobe width $(\mathrm{m})$ & PSLR $(\mathrm{dB})$ & 3dB Mainlobe width $(\mathrm{m})$ & PSLR (dB) \\
\hline 1) $f_{0}=200 \mathrm{MHz}, L_{\phi}=0.1707 \mathrm{~s}$ & 21.4844 & -10.915 & 13.9906 & -13.08 \\
2) $f_{0}=200 \mathrm{MHz}, L_{\phi}=0.6827 \mathrm{~s}$ & 19.7656 & -21.4 & 14.1926 & -22.3193 \\
3) $f_{0}=20 \mathrm{MHz}, L_{\phi}=0.6827 \mathrm{~s}$ & 140.078 & -21.995 & 59.7266 & -17.0983 \\
\hline
\end{tabular}

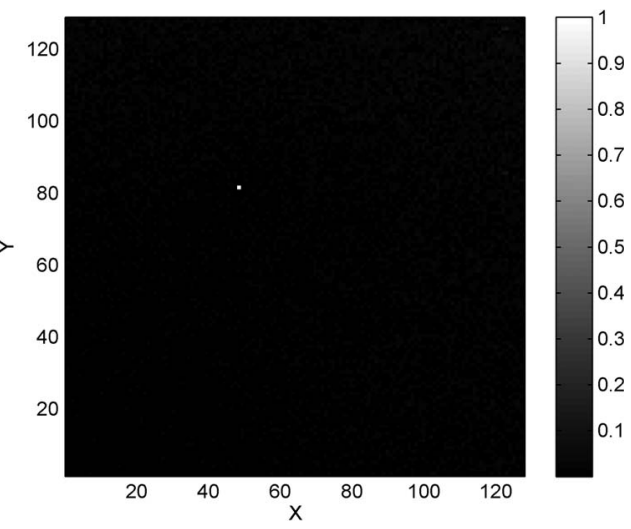

Fig. 10. PSF at $[9.625,12.375,0] \mathrm{km}$ with $16 \tau^{\prime}$ values uniformly spaced in $[0,314.159]$ s using a single cooperative DAB transmitter located at $\mathbf{y}_{0}$ and two receivers traversing the circular flight trajectories $\gamma_{1}(s)$ and $\gamma_{2}(s)$, as shown in Fig. 14.

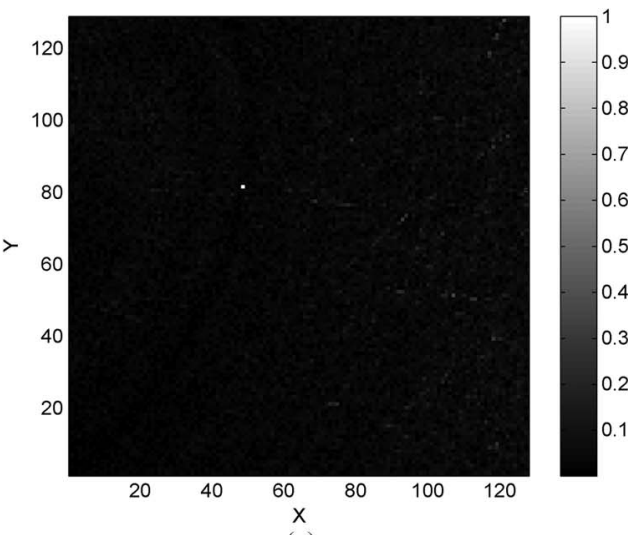

(a)

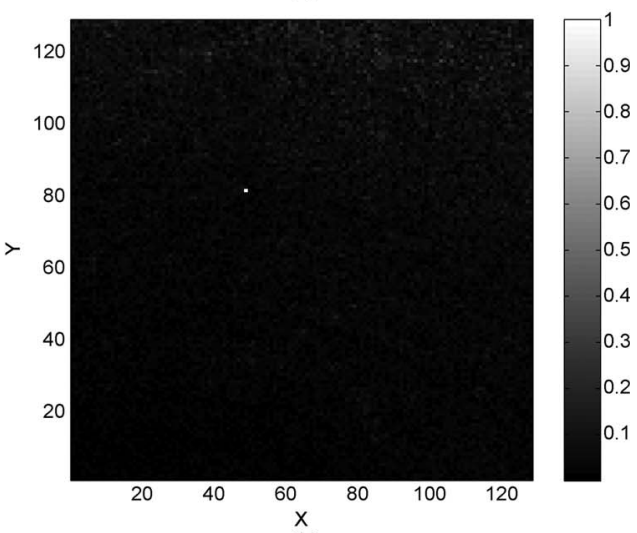

(b)

Fig. 11. PSFs using the vectors in the partial data collection manifolds (a) $\Omega_{12, \tau^{\prime}}=78.54 \mathrm{~s}$ (corresponding to $s=\pi / 2$ ) and (b) $\Omega_{12, \tau^{\prime}}=255.254 \mathrm{~s}$ (corresponding to $s=1.625 \pi$ ) with a single cooperative DAB transmitter located at $\mathbf{y}_{0}$, transmitting a DAB waveform at $f_{0}=200 \mathrm{MHz}$, and two receivers traversing circular flight trajectories $\gamma_{1}(s)$ and $\gamma_{2}(s)$, as shown in Fig. 14. The length of the support of the windowing function is $L_{\phi}=0.3413 \mathrm{~s}$.

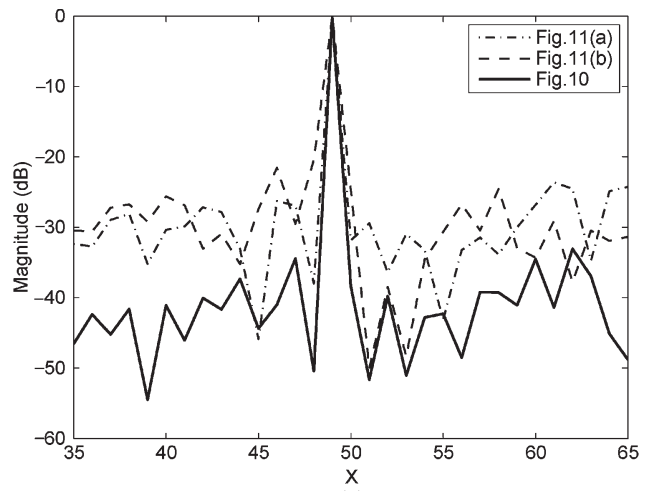

(a)

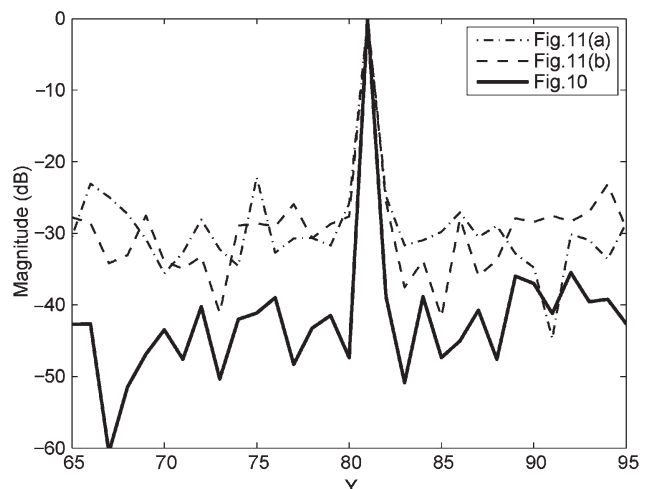

(b)

Fig. 12. $X$ and $Y$ profiles of the reconstructed PSFs shown in Figs. 10 and 11

in Table II that the resolution in the $X$-direction improves moderately, while the resolution in the $Y$-direction remains almost the same.

Comparing Figs. 8 and 9, we see that the quality of the reconstructed PSF degrades due to the decrease in the carrier frequency of the transmitted waveform from 200 to $20 \mathrm{MHz}$ as indicated by the theory. This is also indicated by the spreading of the mainlobe in Fig. 9(b) and (c) and an increase in the 3-dB mainlobe width and a moderate decrease in PSLR as shown in Table II.

To demonstrate the effect of the superposition of images reconstructed for each $\tau^{\prime}$, we reconstructed the PSF for a range of $\tau^{\prime}$ values. For this simulation, we used both cooperative and noncooperative transmitters with the parameters described earlier. Fig. 10 shows the reconstructed PSF, which is the superposition of the images obtained for 16 different $\tau^{\prime}$ values uniformly spaced in $[0,314.159] \mathrm{s}$ for a cooperative transmitter. Fig. 11(a) and (b) shows the reconstructed images corresponding to two different values of $\tau^{\prime}$. The profiles of the reconstructed PSFs in the $X$ - and $Y$-directions are shown in Fig. 12. Table III includes the 3-dB mainlobe width and PSLR of the $X$ and $Y$ profiles. 
TABLE III

Mainlobe Width of 3 dB And PSLR of the Reconstructed PSFs For Demonstrating the Superposition EFFeCt

\begin{tabular}{c|cc|cc}
\hline & \multicolumn{2}{|c|}{ X direction } & \multicolumn{2}{c}{ Y direction } \\
Cases & 3dB Mainlobe width (m) & PSLR (dB) & 3dB Mainlobe width (m) & PSLR (dB) \\
\hline superposition over 16 $\tau^{\prime}$ & 11.4469 & -28.16 & 12.1602 & -27.0965 \\
a fixed $\tau^{\prime}=78.54 \mathrm{~s}$ & 14.9016 & -16.36 & 20.4531 & -22.1385 \\
a fixed $\tau^{\prime}=255.254 \mathrm{~s}$ & 23.0914 & -17.7 & 19.4219 & -12.1805 \\
\hline
\end{tabular}

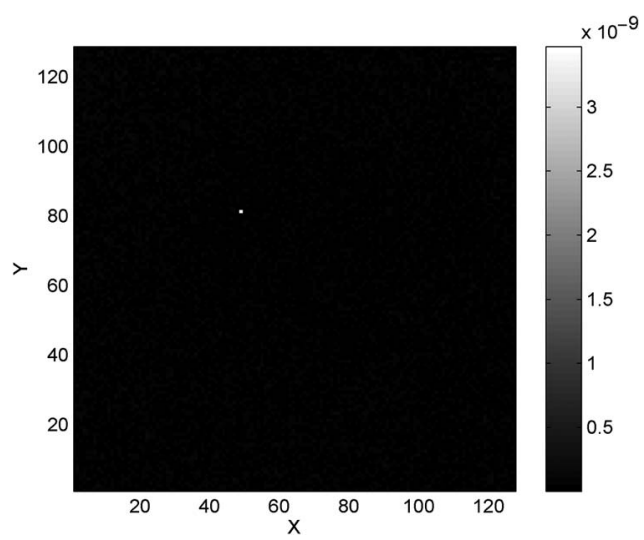

Fig. 13. Reconstructed image for a point target with $16 \tau^{\prime}$ values uniformly spaced in $[0,314.1] \mathrm{s}$ using a single noncooperative transmitter located at $\mathbf{y}_{0}$, transmitting a DAB waveform at $f_{0}=200 \mathrm{MHz}$, and two receivers traversing the circular flight trajectories $\gamma_{1}(s)$ and $\gamma_{2}(s)$ as shown in Fig. 14. The length of the support of the windowing function is $L_{\phi}=0.0853 \mathrm{~s}$.

As expected, since the partial data collection manifold $\Omega_{12, \tau^{\prime}, \boldsymbol{z}}$ for a fixed $\tau^{\prime}$ is significantly smaller than $\cup_{\tau^{\prime}} \Omega_{12, \tau^{\prime}, \boldsymbol{z}}$, the visual quality of the images in Fig. 11 is poorer than that of the superimposed image in Fig. 10. This is also indicated by Fig. 12 and the 3-dB mainlobe widths tabulated in Table III. Furthermore, as shown in Fig. 12 and indicated by the PSLR values in Table III, the superposition suppresses the sidelobes significantly. This can be also observed visually in Fig. 10.

For the case of noncooperative transmitters, we assumed that the scene was illuminated by an isotropic antenna and set $\tilde{R}_{T}=1$. Furthermore, we set $|\mathbf{T}-\mathbf{z}|^{2}=1$ for all $\mathbf{z} \in \mathbb{R}^{3}$ in $G_{i j}$ for the image reconstruction. This can be interpreted as a uninformative prior on the transmitter antenna beam patterns and the transmitter-related geometric spreading factors.

The reconstructed PSF for the case of a single noncooperative transmitter is shown in Fig. 13. Without the transmitterrelated geometric spreading factors in the reconstruction formula, the strength of the target appears weak because of the range-dependent attenuation. If a priori information on the rough location of the transmitters is available, this rangedependent attenuation can be easily corrected by rescaling the correlated received signal.

\section{B. Numerical Simulations for Multiple Point Targets}

In accordance with the incoherent field approximation, we used the following multiple-point-target model for the scene reflectivity:

$$
\rho(\boldsymbol{z})=\sum_{l=1}^{L} g_{l} \delta\left(\boldsymbol{z}-\boldsymbol{z}_{l}\right)
$$

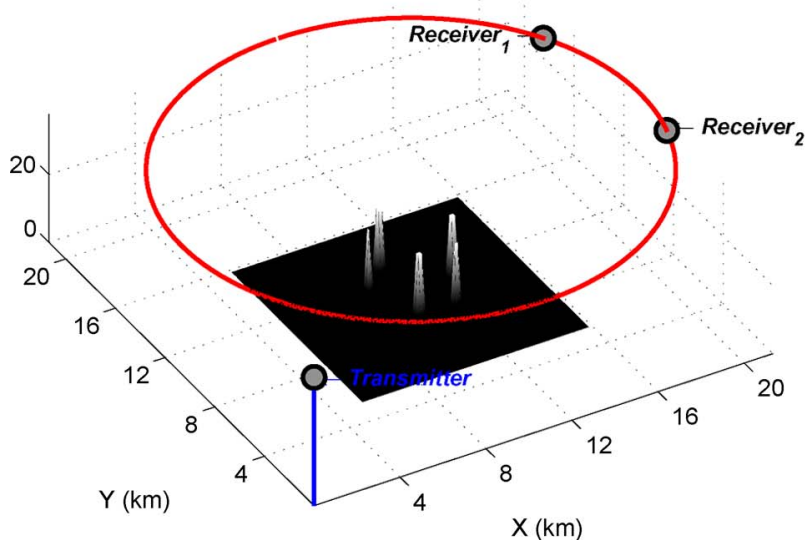

(a)

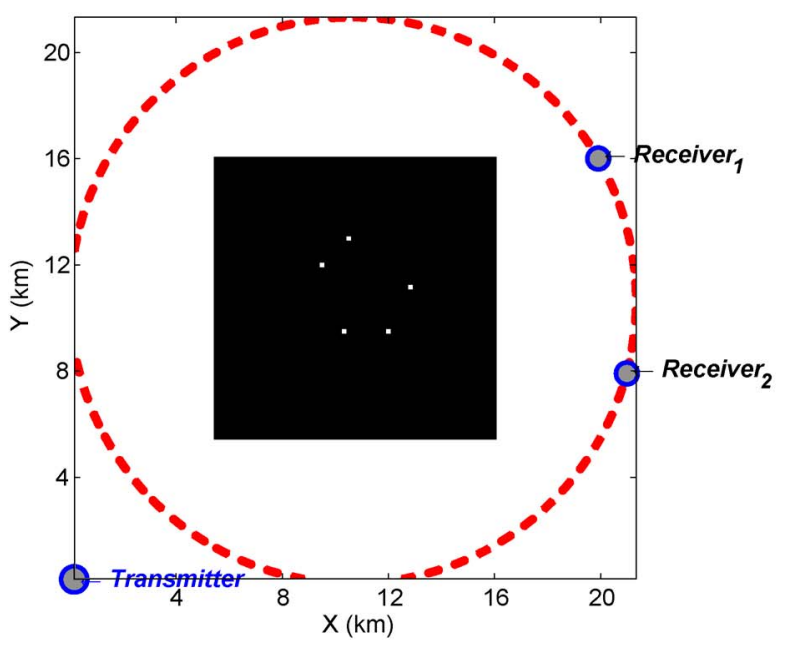

(b)

Fig. 14. (a) 3-D and (b) 2-D views of the scene with multiple point targets, illuminated by a single DAB transmitter located at $\mathbf{y}_{0}=(0,0,6.5) \mathrm{km}$ and the circular receiver trajectory $\gamma_{C}(s)=$ $(11+11 \cos (s), 11+11 \sin (s), 6.5) \mathrm{km}$, as shown by the red solid line. At a certain time instant, two receivers are located at the positions shown in the figure.

where $g_{l}, l=1, \ldots, L$, are independent Gaussian random variables with mean $\mu_{l}$ and variance $\sigma_{l}^{2}$. The corresponding scene radiance is given by

$$
R_{\rho}(\boldsymbol{z})=E\left[\rho(\boldsymbol{z}) \rho^{*}(\boldsymbol{z})\right]=\sum_{l}\left(\mu_{l}^{2}+\sigma_{l}^{2}\right) \delta\left(\boldsymbol{z}-\boldsymbol{z}_{l}\right) .
$$

In our simulations, we considered a deterministic reflectivity and set $\sigma_{l}^{2}=1$. We used $L=5$ and approximated the Diracdelta functions in (69) by square target reflectors of size $344 \times 344 \mathrm{~m}^{2}$, each having a unit reflectivity, i.e., $\mu_{l}=1$, $l=1, \ldots, 5$. Fig. 14(a) and (b) shows the scene with targets, receiver trajectories, and the transmitter antenna location. 


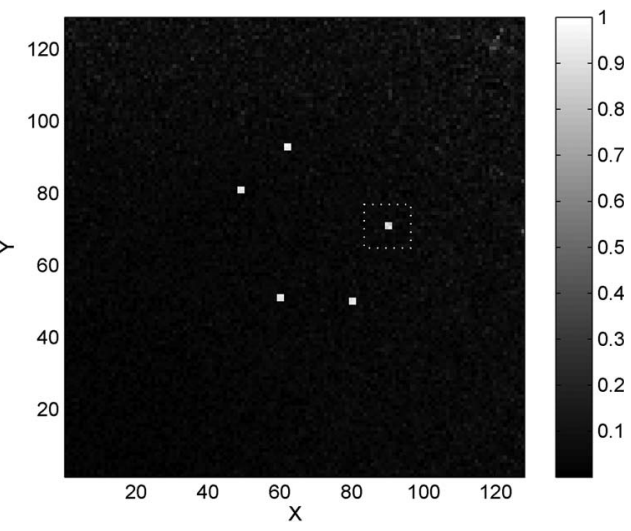

Fig. 15. Reconstructed image for multiple point targets with $16 \tau^{\prime}$ values uniformly spaced in $[0,314.159]$ s using a single cooperative transmitter located at $\mathbf{y}_{0}$ and two receivers traversing the circular flight trajectories $\gamma_{1}(s)$ and $\gamma_{2}(s)$ as shown in Fig. 14 .

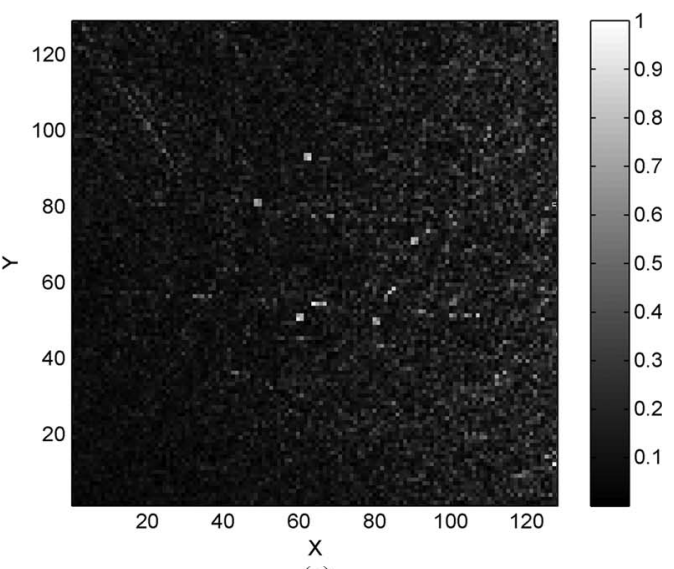

(a)

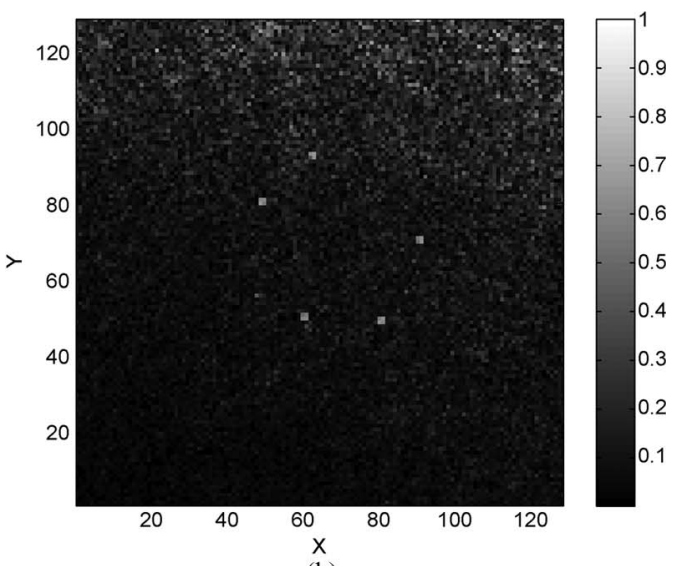

(b)

Fig. 16. Images reconstructed for multiple point targets using the vectors in the partial data collection manifolds (a) $\Omega_{12, \tau^{\prime}=78.54 \mathrm{~s}}$ (corresponding to $s=$ $\pi / 2$ ) and (b) $\Omega_{12, \tau^{\prime}}=255.254 \mathrm{~s}$ (corresponding to $s=1.625 \pi$ ), with a single cooperative transmitter located at $\mathbf{y}_{0}$ and two receivers traversing circular flight trajectories $\gamma_{1}(s)$ and $\gamma_{2}(s)$ as shown in Fig. 14.

1) Cooperative Transmitters: For the case of a cooperative transmitter, the reconstructed image is shown in Fig. 15. The two images corresponding to the fixed $\tau^{\prime}$ values are shown in Fig. 16(a) and (b). Fig. 17 shows the profiles in the $X$ - and $Y$-directions of the reconstructed point target indicated by the white square in Fig. 15.

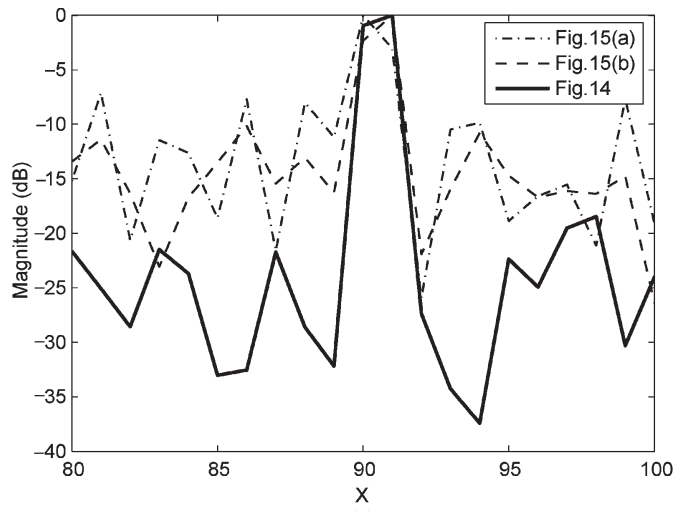

(a)

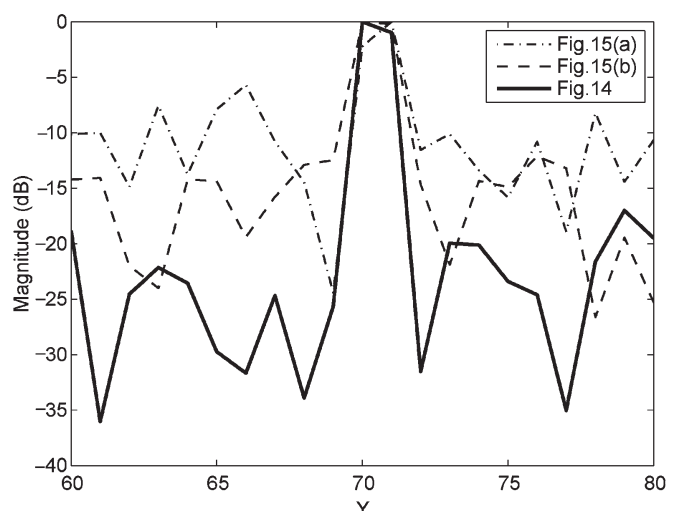

(b)

Fig. 17. $X$ and $Y$ profiles of one of the reconstructed targets (indicated by the white dashed square) shown in Fig. 15.

As expected, due to a smaller data collection manifold, the quality of the reconstructed images for fixed $\tau^{\prime}$ values, as shown in Fig. 16(a) and (b), is poorer than that of the image obtained by the superposition of all the images reconstructed for a range of $\tau^{\prime}$ values, $\tau^{\prime} \in[0,314.159] \mathrm{s}$, as shown in Fig. 15. We observe that some of the targets do not appear as sharp as the ones in Fig. 15. The analysis of the $X$ and $Y$ profiles of one of the reconstructed point targets shows the improvement of the reconstruction more clearly. As can be seen in Fig. 17, the mainlobe width becomes narrower, and the level of the sidelobes is significantly reduced in the superimposed image, which results in better visual quality reconstruction, as shown in Fig. 15.

2) Noncooperative Transmitters: For the case of noncooperative transmitters, we assumed that the scene was illuminated by an isotropic antenna and set $\tilde{R}_{T}=1$. Furthermore, we set $|\mathbf{T}-\mathbf{z}|^{2}=1$ for all $\mathbf{z} \in \mathbb{R}^{3}$ in $G_{i j}$ for the image reconstruction. This can be interpreted as a uninformative prior on the transmitter antenna beam patterns and the transmitter-related geometric spreading factors.

Fig. 18 shows the reconstructed image for multiple point targets using a noncooperative transmitter. Since the location of the transmitter was assumed to be unknown, the received signal was not compensated for the transmitter-related geometric spreading factors. As a result, the scatterers closer to the transmitter appear brighter in the reconstructed image than those that are further away from the transmitter. Table IV tabulates the amplitudes of each target. The amplitude is calculated by averaging the values of the pixels where the target is located. 


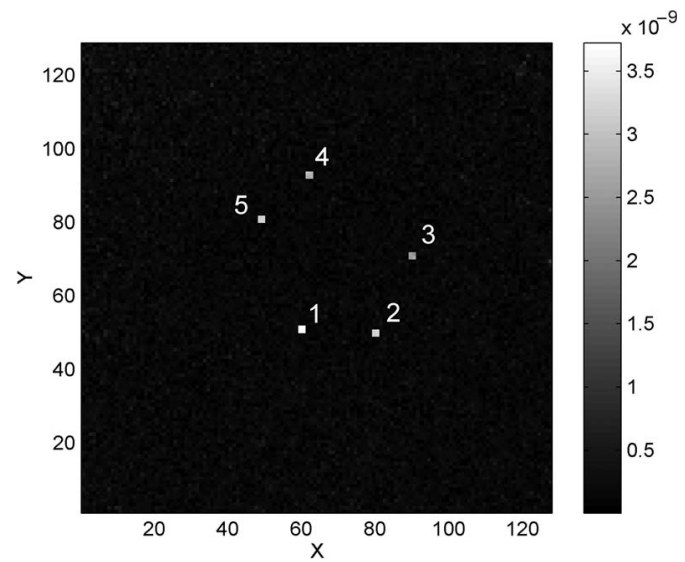

Fig. 18. Reconstructed image for multiple point targets with $16 \tau^{\prime}$ values uniformly sampled in $[0,314.159]$ s using a single noncooperative transmitter located at $\mathbf{y}_{0}$ and two receivers traversing the circular flight trajectories $\gamma_{1}(s)$ and $\gamma_{2}(s)$ as shown in Fig. 14.

TABLE IV

AMPLITUDES OF THE RECONSTRUCTED TARGETS

\begin{tabular}{c|c}
\hline Target number & Amplitude $(1 \mathrm{e}-9)$ \\
\hline 1 & 3.3913 \\
2 & 3.1853 \\
3 & 2.555 \\
4 & 2.8396 \\
5 & 3.1878 \\
\hline
\end{tabular}

\section{CONCLUSION}

We have introduced a novel SAR modality, which we referred to as the Doppler-hitchhiker, for synthetic aperture imaging. Doppler-hitchhiker uses ultranarrowband waveforms of opportunity transmitted by radio, television, cell phone, etc., stations and one or more receivers traversing arbitrary trajectories.

Doppler-hitchhiker performs image formation in two steps: In the first step, the received signal by each receiver is partitioned into windows and then scaled, translated, and correlated with the received data in other windows measured by the same and/or different receivers. The second step involves high-frequency analysis of the resulting forward model and the filtered backpropagation of the correlated signals onto the hitchhiker iso-Doppler contours to form an image. Our resolution analysis shows that the spread of the hitchhiker iso-Doppler curves is directly related to the Doppler ambiguity function of the transmitted waveforms. The Doppler ambiguity functions of the $\mathrm{CW}$ or ultranarrowband waveforms have narrow peak resulting in narrow hitchhiker iso-Doppler contours. Thus, intuitively speaking, backprojecting onto these curves results in high-resolution imaging. Our analysis shows that the resolution of the reconstructed image is determined primarily by the temporal duration and frequency of the transmitted waveforms, which is consistent with the ambiguity theory of the $\mathrm{CW}$ or ultranarrowband waveforms.

We used microlocal techniques to backproject the correlated signals onto the hitchhiker iso-Doppler contours. Our reconstruction is a filtered-backprojection type and has the desirable property of preserving the visible edges of the scene. We presented a high-frequency analysis of the PSF of our imaging operator and showed that the filtered-backprojection operator recovers the scene at the intersection of the hitchhiker isoDoppler and hitchhiker iso-Doppler-rate contours. We note that the our image reconstruction technique easily accommodates arbitrary trajectories, nonflat topography, and system-related parameters. Furthermore, it is an analytic technique that can be made computationally efficient using the fast-backprojection algorithms [22]. We demonstrated the performance of our imaging method in numerical simulations using the DAB signals as the illumination source of opportunity and verified the theoretical results.

While our image formation was performed in a deterministic setting, following the methodology that we introduced in [20], [30], [33], and [34], we can show that the correlation of scaled and delayed measurements is an optimal detection scheme for a point target in the presence of additive white Gaussian noise. This result, as well as imaging in the presence of more general noise models and performance analysis, is described in our work [21].

Although we presented our approach specifically for passive SAR, our method is also applicable to other passive imaging problems, such as passive geophysical or acoustic imaging.

\section{APPENDIX A \\ DERIVATIONS RELATED TO THE DSAH ISO-DOPPLER-RATE CONTOURS}

Substituting (18) into

$$
\begin{aligned}
\partial_{\tau}\left(\omega_{0} t\left[1-\left(\boldsymbol{\gamma}_{j} \widehat{(\tau)}-\mathbf{z}\right) \cdot \dot{\gamma}_{j}(\tau) / c_{0}\right]\right. \\
{\left.\left[S_{i j}\left(\tau^{\prime}, \tau, \boldsymbol{z}^{\prime}\right)-S_{i j}\left(\tau^{\prime}, \tau, \boldsymbol{z}\right)\right]\right)=0 }
\end{aligned}
$$

we reduce (70) to

$$
\begin{aligned}
-\omega_{0} t\left[1-\left(\gamma_{i} \widehat{\left(\tau^{\prime}\right)-} \mathbf{z}^{\prime}\right) \cdot \dot{\gamma}_{i}\left(\tau^{\prime}\right) / c_{0}\right] \\
\times \underbrace{}_{\Delta} \underbrace{}_{\partial_{\tau}\left[\frac{1-\left(\gamma_{j} \widehat{(\tau)}-\mathbf{z}\right) \cdot \dot{\gamma}_{j}(\tau) / c_{0}}{1-\left(\gamma_{j} \widehat{(\tau)}-\mathbf{z}^{\prime}\right) \cdot \dot{\gamma}_{j}(\tau) / c_{0}}\right]}=0 .
\end{aligned}
$$

We now express $\Delta$ as

$$
\begin{aligned}
& \partial_{\tau}\left[\frac{1-\left(\widehat{\gamma_{j}} \widehat{(\tau)}-\mathbf{z}\right) \cdot \dot{\gamma}_{j}(\tau) / c_{0}}{1-\left(\gamma_{j} \widehat{(\tau)}-\mathbf{z}^{\prime}\right) \cdot \dot{\gamma}_{j}(\tau) / c_{0}}\right] \\
& =-\frac{1-\left(\gamma_{j} \widehat{(\tau)}-\mathbf{z}\right) \cdot \dot{\gamma}_{j}(\tau) / c_{0}}{\left[1-\left(\gamma_{j} \widehat{(\tau)-} \mathbf{z}^{\prime}\right) \cdot \dot{\gamma}_{j}(\tau) / c_{0}\right]^{2}} \\
& \times \partial_{\tau}\left[1-\left(\gamma_{j} \widehat{(\tau)-} \mathbf{z}^{\prime}\right) \cdot \dot{\gamma}_{j}(\tau) / c_{0}\right] \\
& +\frac{1}{1-\left(\gamma_{j} \widehat{(\tau)-} \mathbf{z}^{\prime}\right) \cdot \dot{\gamma}_{j}(\tau) / c_{0}} \\
& \times \partial_{\tau}\left[1-\left(\gamma_{j} \widehat{(\tau)}-\mathbf{z}\right) \cdot \dot{\gamma}_{j}(\tau) / c_{0}\right] \text {. }
\end{aligned}
$$


Introducing (72) into (71), we obtain

$$
\begin{aligned}
\frac{\partial_{\tau}\left[1-\left(\widehat{\gamma_{j}} \widehat{(\tau)}-\mathbf{z}\right) \cdot \dot{\gamma}_{j}(\tau) / c_{0}\right]}{1-\left(\gamma_{j}(\tau)-\mathbf{z}\right)} \cdot \dot{\gamma}_{j}(\tau) / c_{0} \\
=\frac{\partial_{\tau}\left[1-\left(\gamma_{j} \widehat{(\tau)-} \mathbf{z}^{\prime}\right) \cdot \dot{\gamma}_{j}(\tau) / c_{0}\right]}{1-\left(\gamma_{j} \widehat{(\tau)-} \mathbf{z}^{\prime}\right) \cdot \dot{\gamma}_{j}(\tau) / c_{0}} .
\end{aligned}
$$

Let us focus on the numerators on both sides of (73). Using the notations $\gamma_{j}=\left[\gamma_{j}^{1}, \gamma_{j}^{2}, \gamma_{j}^{3}\right]^{T}$ and $\dot{\gamma}_{j}=\left[\dot{\gamma}_{j}^{1}, \dot{\gamma}_{j}^{2}, \dot{\gamma}_{j}^{3}\right]^{T}$, the partial derivative of the numerator on the left-hand side of (73) with respect to $\tau$ can be expressed as

$$
\left.\begin{array}{rl}
\partial_{\tau}[1 & -\left(\boldsymbol{\gamma}_{j} \widehat{\left.(\tau)-\mathbf{z}) \cdot \dot{\gamma}_{j}(\tau) / c_{0}\right]}\right. \\
= & \partial_{\tau} \frac{-1}{c_{0}\left|\gamma_{j}(\tau)-\mathbf{z}\right|} \\
& \times\left[\left(\gamma_{j}^{1}(\tau)-z_{1}\right) \dot{\gamma}_{j}^{1}(\tau)\right. \\
& \left.+\left(\gamma_{j}^{2}(\tau)-z_{2}\right) \dot{\gamma}_{j}^{2}(\tau)+\left(\gamma_{j}^{3}(\tau)-z_{3}\right) \dot{\gamma}_{j}^{3}(\tau)\right] \\
= & \left.\frac{1}{c_{0}\left|\gamma_{j}(\tau)-\mathbf{z}\right|^{2}} \frac{\gamma_{j}(\tau)-\mathbf{z}}{\left|\gamma_{j}(\tau)-\mathbf{z}\right|} \cdot \dot{\gamma}_{j}(\tau)\right] \\
& \times\left[\left(\gamma_{j}(\tau)-\mathbf{z}\right) \cdot \dot{\gamma}_{j}(\tau)\right]-\frac{1}{c_{0}\left|\gamma_{j}(\tau)-\mathbf{z}\right|} \\
& \times\left[\left(\dot{\gamma}_{j}^{1}(\tau)\right)^{2}+\left(\gamma_{j}^{1}(\tau)-z_{1}\right) \ddot{\gamma}_{j}^{1}(\tau)\right. \\
= & \frac{-1}{c_{0}}[\frac{1}{\left|\gamma_{j}(\tau)-\mathbf{z}\right|}\left|\dot{\gamma}_{j, \perp}(\tau, \mathbf{z})\right|^{2}+\underbrace{}_{j}\left(\gamma_{j}(\tau)-\mathbf{z}\right) \cdot \ddot{\gamma}_{j}(\tau)
\end{array}\right]
$$

where

$$
\dot{\gamma}_{j, \perp}(\tau, \mathbf{z})=\dot{\gamma}_{j}(\tau)-\left(\gamma_{j} \widehat{(\tau)}-\mathbf{z}\right)\left(\left(\gamma_{j} \widehat{(\tau)}-\mathbf{z}\right) \cdot \dot{\gamma}_{j}(\tau)\right)
$$

denotes the projection of the receiver velocity $\dot{\gamma}_{j}(\tau)$ onto the plane whose normal direction is along $\widehat{\gamma_{j}} \widehat{(\tau)}-\mathbf{z}$ and $a_{j}(\tau, \mathbf{z})$ denotes the projection of the receiver acceleration $\ddot{\gamma}_{j}(\tau)$ along $\gamma_{j} \widehat{(\tau)}-\mathbf{z}$. We see that the summation of the two terms in the square bracket of (74) is the total radial acceleration of the $j$ th receiver evaluated at $\tau$. We denote these two terms by $a_{j}(\tau, \mathbf{z})$, i.e.,

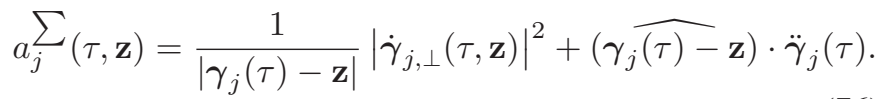

Introducing (74) into (73), we obtain

$$
\frac{a_{j}^{\sum_{(\tau, \mathbf{z})}}}{1-\left(\gamma_{j} \widehat{(\tau)}-\mathbf{z}\right) \cdot \dot{\gamma}_{j}(\tau) / c_{0}}=\frac{a_{j}^{\sum_{j}}\left(\tau, \mathbf{z}^{\prime}\right)}{1-\left(\gamma_{j} \widehat{(\tau)-} \mathbf{z}^{\prime}\right) \cdot \dot{\gamma}_{j}(\tau) / c_{0}} .
$$

\section{APPENDIX B}

DERIVATIONS RELATED TO THE DSAH

DATA COLlECtion MANifold

Introducing (18) in (45)

$\boldsymbol{\Xi}_{i j}\left(\tau^{\prime}, \tau, \boldsymbol{z}\right)=\omega_{0}\left[1-\left(\widehat{\gamma_{j}(\tau)-} \mathbf{z}\right) \cdot \dot{\gamma}_{j}(\tau) / c_{0}\right] \nabla_{\boldsymbol{z}} S_{i j}\left(\tau^{\prime}, \tau, \boldsymbol{z}\right)$

we obtain

$$
\begin{aligned}
& \boldsymbol{\Xi}_{i j}\left(\tau^{\prime}, \tau, \boldsymbol{z}\right)=\omega_{0}\left[\nabla_{\mathbf{z}}\left(1-\left(\boldsymbol{\gamma}_{i} \widehat{\left(\tau^{\prime}\right)}-\mathbf{z}\right) \cdot \dot{\gamma}_{i}\left(\tau^{\prime}\right) / c_{0}\right)\right. \\
& -\frac{1-\left(\gamma_{i} \widehat{\left(\tau^{\prime}\right)}-\mathbf{z}\right) \cdot \dot{\gamma}_{i}\left(\tau^{\prime}\right) / c_{0}}{1-\left(\gamma_{j} \widehat{(\tau)}-\mathbf{z}\right) \cdot \dot{\gamma}_{j}(\tau) / c_{0}} \\
& \left.\nabla_{\mathbf{z}}\left(1-\left(\gamma_{j} \widehat{(\tau)}-\mathbf{z}\right) \cdot \dot{\gamma}_{j}(\tau) / c_{0}\right)\right]
\end{aligned}
$$

where $\nabla_{\mathbf{z}}=\left[\partial_{z_{1}}, \partial_{z_{2}}\right]^{T}$.

Defining $\mu=S_{i j}\left(\tau^{\prime}, \tau, \boldsymbol{z}\right)$, (79) can be expressed as

$$
\begin{aligned}
& \boldsymbol{\Xi}\left(\tau^{\prime}, \tau, \boldsymbol{z}\right)=\omega_{0}\left[\nabla_{\mathbf{z}}\left(1-\left(\boldsymbol{\gamma}_{i} \widehat{\left(\tau^{\prime}\right)}-\mathbf{z}\right) \cdot \dot{\gamma}_{i}\left(\tau^{\prime}\right) / c_{0}\right)\right. \\
& \left.-\mu \nabla_{\mathbf{z}}\left(1-\left(\gamma_{j} \widehat{(\tau)}-\mathbf{z}\right) \cdot \dot{\gamma}_{j}(\tau) / c_{0}\right)\right] \\
& =\frac{\omega_{0}}{c_{0}}\left[\mu \nabla_{\mathbf{z}}\left(\gamma_{j} \widehat{(\tau)}-\mathbf{z} \cdot \dot{\gamma}_{j}(\tau)\right)\right. \\
& \left.-\nabla_{\mathbf{z}}\left(\gamma_{i} \widehat{\left(\tau^{\prime}\right)}-\mathbf{z} \cdot \dot{\gamma}_{i}\left(\tau^{\prime}\right)\right)\right] .
\end{aligned}
$$

Let us write

$$
\begin{aligned}
& \left(\boldsymbol{\gamma}_{i} \widehat{\left(\tau^{\prime}\right)}-\mathbf{z}\right) \cdot \dot{\gamma}_{i}\left(\tau^{\prime}\right)=\frac{1}{\left|\gamma_{i}\left(\tau^{\prime}\right)-\mathbf{z}\right|}\left[\left(\gamma_{i}^{1}\left(\tau^{\prime}\right)-z_{1}\right) \dot{\gamma}_{i}^{1}\left(\tau^{\prime}\right)\right. \\
& \left.+\left(\gamma_{i}^{2}\left(\tau^{\prime}\right)-z_{2}\right) \dot{\gamma}_{i}^{2}\left(\tau^{\prime}\right)+\left(\gamma_{i}^{3}\left(\tau^{\prime}\right)-\psi\left(z_{1}, z_{2}\right)\right) \dot{\gamma}_{i}^{3}\left(\tau^{\prime}\right)\right]
\end{aligned}
$$

where $\gamma_{i}=\left[\gamma_{i}^{1}, \gamma_{i}^{2}, \gamma_{i}^{3}\right]^{T}$ and $\dot{\gamma}_{i}=\left[\dot{\gamma}_{i}^{1}, \dot{\gamma}_{i}^{2}, \dot{\gamma}_{i}^{3}\right]^{T}$. Calculating the first-order partial derivative of (81) with respect to $z_{1}$, 
we obtain

$$
\begin{aligned}
& \frac{\partial\left(\boldsymbol{\gamma}_{i} \widehat{\left(\tau^{\prime}\right)}-\mathbf{z}\right) \cdot \dot{\gamma}_{i}\left(\tau^{\prime}\right)}{\partial z_{1}} \\
&=\frac{-1}{\left|\gamma_{i}\left(\tau^{\prime}\right)-\mathbf{z}\right|^{2}}\left[\frac{-\left(\gamma_{i}^{1}\left(\tau^{\prime}\right)-z_{1}\right)-\frac{\partial \psi}{\partial z_{1}}\left(\gamma_{i}^{3}\left(\tau^{\prime}\right)-\psi\left(z_{1}, z_{2}\right)\right)}{\left|\gamma_{i}\left(\tau^{\prime}\right)-\mathbf{z}\right|}\right. \\
& \\
&\left.\quad \times\left(\left(\gamma_{i}\left(\tau^{\prime}\right)-\mathbf{z}\right) \cdot \dot{\gamma}_{i}\left(\tau^{\prime}\right)\right)\right] \\
&+\frac{1}{\left|\gamma_{i}\left(\tau^{\prime}\right)-\mathbf{z}\right|}\left[-\dot{\gamma}_{i}^{1}\left(\tau^{\prime}\right)-\frac{\partial \psi}{\partial z_{1}} \dot{\gamma}_{i}^{3}\left(\tau^{\prime}\right)\right] .
\end{aligned}
$$

Similarly, we obtain the partial differential of (81) with respect to $z_{2}$.

Let

$$
D \psi(\boldsymbol{z})=\left[\begin{array}{lll}
1 & 0 & \partial \psi(\boldsymbol{z}) / \partial z_{1} \\
0 & 1 & \partial \psi(\boldsymbol{z}) / \partial z_{2}
\end{array}\right] .
$$

Then, using (82), we have

$$
\begin{aligned}
& \nabla_{\mathbf{z}}\left(\boldsymbol{\gamma}_{i} \widehat{\left(\tau^{\prime}\right)}-\mathbf{z}\right) \cdot \dot{\gamma}_{i}\left(\tau^{\prime}\right)=\frac{-1}{\left|\gamma_{i}\left(\tau^{\prime}\right)-\mathbf{z}\right|} \\
& \times D \psi(\boldsymbol{z}) \cdot[\underbrace{\dot{\gamma}_{i}\left(\tau^{\prime}\right)-\left(\gamma_{i} \widehat{\left(\tau^{\prime}\right)}-\mathbf{z}\right)\left(\left(\gamma_{i} \widehat{\left(\tau^{\prime}\right)}-\mathbf{z}\right) \cdot \dot{\gamma}_{i}\left(\tau^{\prime}\right)\right)}_{\dot{\gamma}_{i, \perp}\left(\tau^{\prime}\right)}] .
\end{aligned}
$$

Similarly, we obtain

$$
\begin{aligned}
& \nabla_{\mathbf{z}}\left(\widehat{\gamma_{j}(\tau)-} \mathbf{z}\right) \cdot \dot{\gamma}_{j}(\tau)=\frac{-1}{\left|\gamma_{j}(\tau)-\mathbf{z}\right|} \\
& \quad \times D \psi(\boldsymbol{z}) \cdot[\underbrace{\dot{\gamma}_{j}(\tau)-\left(\gamma_{j} \widehat{(\tau)-} \mathbf{z}\right)\left(\widehat{\left.\left(\gamma_{j}(\tau)-\mathbf{z}\right) \cdot \dot{\gamma}_{j}(\tau)\right)}\right.}_{\dot{\gamma}_{j, \perp}(\tau)}] .
\end{aligned}
$$

Note that $\dot{\gamma}_{i, \perp}\left(\tau^{\prime}\right)$ and $\dot{\gamma}_{j, \perp}(\tau)$ are the projections of the receiver velocities $\dot{\gamma}_{i}\left(\tau^{\prime}\right)$ and $\dot{\gamma}_{j}(\tau)$ onto the planes perpendicular to the directions $\gamma_{i} \widehat{\left(\tau^{\prime}\right)}-\mathbf{z}$ and $\gamma_{j} \widehat{(\tau)}-\mathbf{z}$, respectively.

Substituting (84) and (85) into (80), we obtain

$$
\begin{aligned}
& \boldsymbol{\Xi}_{i j}\left(\tau^{\prime}, \tau, \boldsymbol{z}\right)=\frac{\omega_{0}}{c_{0}} D \psi(\boldsymbol{z}) \\
& \cdot\left[\frac{1}{\left|\boldsymbol{\gamma}_{i}\left(\tau^{\prime}\right)-\mathbf{z}\right|} \dot{\gamma}_{i, \perp}\left(\tau^{\prime}\right)-\frac{\mu}{\left|\gamma_{j}(\tau)-\mathbf{z}\right|} \dot{\gamma}_{j, \perp}(\tau)\right] .
\end{aligned}
$$

\section{REFERENCES}

[1] H. D. Griffiths and N. R. W. Long, "Television-based bistatic radar," Proc. Inst. Elect. Eng.—Radar Sonar Navig., vol. 133, no. 7, pp. 649-657, Dec. 1986.

[2] V. Koch and R. Westphal, "New approach to a multistatic passive radar sensor for air/space defense," IEEE Aerosp. Electron. Syst. Mag., vol. 10, no. 11, pp. 24-32, Nov. 1995.

[3] H. D. Griffiths and C. J. Baker, "Passive coherent location radar systems. Part 1: Performance prediction," Proc. Inst. Elect. Eng.-Radar Sonar Navig., vol. 152, no. 3, pp. 153-159, Jun. 2005
[4] C. J. Baker, H. D. Griffiths, and I. Papoutsis, "Passive coherent location radar systems. Part 2: Waveform properties," Proc. Inst. Elect. Eng.-Radar Sonar Navig., vol. 152, no. 3, pp. 160-168, Jun. 2005.

[5] D. Poullin, "Passive detection using digital broadcasters (DAB, DVB) with COFDM modulation," Proc. Inst. Elect. Eng.-Radar Sonar Navig., vol. 152, no. 3, pp. 143-152, Jun. 2005.

[6] R. Pollard, The role of passive radar sensors for air traffic control, Inst. Eng. Technol. Semin. Future Civil Radar, 2006.

[7] D. K. P. Tan, H. Sun, Y. Lu, M. Lesturgie, and H. L. Chan, "Passive radar using global system for mobile communication signal: Theory, implementation and measurements," Proc. Inst. Elect. Eng.—Radar Sonar Navig., vol. 152, no. 3, pp. 116-123, Jun. 2005.

[8] P. E. Howland, D. Maksimiuk, and G. Reitsma, "Fm radio based bistatic radar," Proc. Inst. Elect. Eng.—Radar Sonar Navig., vol. 152, no. 3, pp. 107-115, Jun. 2005.

[9] C. Coleman and H. Yardley, "Passive bistatic radar based on target illuminations by digital audio broadcasting," IET Radar Sonar Navig., vol. 2, no. 5, pp. 366-375, Oct. 2008

[10] H. Guo, K. Woodbridge, and C. J. Baker, "Evaluation of WiFi beacon transmissions for wireless based passive radar," in Proc. IEEE Radar Conf., Roma, Italy, May 2008, pp. 1-6.

[11] D. W. O'Hagan and C. J. Baker, "Passive Bistatic Radar (PBR) using FM radio illuminators of opportunity," in Proc. IEEE Radar Conf., Roma, Italy, May 2008, pp. 1-6.

[12] J. Homer, K. Kubik, B. Mojarrabi, I. Longstaff, E. Donskoi, and M. Cherniakov, "Passive bistatic radar sensing with LEOS based transmitters," in Proc. IEEE Int. Geosci. Remote Sens. Symp., Jun. 2002, vol. 1, pp. 438-440.

[13] K. Chetty, K. Woodbridge, H. Guo, and G. E. Smith, "Passive bistatic WiMAX radar for marine surveillance," in Proc. IEEE Radar Conf., Washington, DC, May 2010, pp. 188-193.

[14] P. Falcone, F. Colone, C. Bongioanni, and P. Lombardo, "Experimental results for OFDM WiFi-based passive bistatic radar," in Proc. IEEE Radar Conf., Washington, DC, May 2010, pp. 516-521.

[15] H. A. Harms, L. M. Davis, and J. Palmer, "Understanding the signal structure in DVB-T signals for passive radar detection," in Proc. IEEE Radar Conf., Washington, DC, May 2010, pp. 532-537.

[16] J. M. Christiansen and K. E. Olsen, "Range and Doppler walk in DVB-T based passive bistatic radar," in Proc. IEEE Radar Conf., Washington, DC, May 2010, pp. 620-626.

[17] G. Li, J. Xu, Y. N. Peng, and X. G. Xia, "Bistatic linear array SAR for moving target detection, location and imaging with two passive airborne radars," IEEE Trans. Geosci. Remote Sens., vol. 45, no. 3, pp. 554-565, Mar. 2007.

[18] M. Antoniou, M. Cherniakov, and C. Hu, "Space-surface bistatic SAR image formation algorithms," IEEE Trans. Geosci. Remote Sens., vol. 47, no. 6, pp. 1827-1843, Jun. 2009.

[19] C. E. Yarman and B. Yazıcı, "Synthetic aperture hitchhiker imaging," IEEE Trans. Imaging Process., vol. 17, no. 11, pp. 2156-2173, Nov. 2008

[20] L. Wang, I. Y. Son, and B. Yazıc1, "Passive imaging using distributed apertures in multiple scattering environments," Inverse Problems, vol. 26, no. 6, p. 065 002, Jun. 2010.

[21] L. Wang and B. Yazıc1, "Passive imaging of moving targets using distributed apertures," Inverse Problems, submitted for publication.

[22] S. Nilsson, "Application of fast backprojection techniques for some inverse problems of integral geometry," Ph.D. dissertation, Linköping Studies in Science and Technology, Linköping, Sweden, 1997, dissertation No. 499

[23] C. Nolan and M. Cheney, "Synthetic aperture inversion for arbitrary flight paths and non-flat topography," IEEE Trans. Image Process., vol. 12, no. 9, pp. 1035-1043, Sep. 2003.

[24] H. Barrett and K. Myers, Foundations of Image Science. Hoboken, NJ: Wiley-Interscience, 2004.

[25] F. Treves, Introduction to Pseudodifferential and Fourier Integral Operators, volumes I and II. New York: Plenum, 1980.

[26] F. Natterer and F. Wübbeling, Mathematical Methods in Image Reconstruction. Philadelphia, PA: SIAM, 2001.

[27] N. Bleistein and R. Handelsman, Asymptotic Expansions of Integrals. New York: Dover, 1986.

[28] A. Grigis and J. Sjöstrand, Microlocal Analysis for Differential Operators: An Introduction, ser. London Mathematical Society Lecture Note Series. Cambridge, U.K.: Cambridge Univ. Press, 1994.

[29] V. Guillemin and S. Sternberg, Geometric Asymptotics. Providence, RI: AMS, 1979.

[30] B. Yazici, M. Cheney, and C. Yarman, "Synthetic-aperture inversion in the presence of noise and clutter," Inverse Problems, vol. 22, no. 5, pp. 17051729, Oct. 2006. 
[31] C. J. Nolan and M. Cheney, "Synthetic aperture inversion," Inverse Problems, vol. 18, no. 1, pp. 221-236, Feb. 2002.

[32] E. Quinto, "Singularities of the X-ray transform and limited data tomography in $r^{2}$ and $r^{3}$," SIAM J. Math. Anal., vol. 24, no. 5, pp. 1215-1225, Sep. 1993.

[33] I. Y. Son, T. Varslot, C. E. Yarman, A. Pezeshki, B. Yazici, and M. Cheney, "Radar detection using sparsely distributed apertures in urban environment," in Proc. SPIE Def., Secur. Sens. Conf., Orlando, FL, Apr. 2007, vol. 6567, pp. 65 671Q-1-65 671Q-8.

[34] K. Voccola, B. Yazici, M. Cheney, and M. Ferrara, "On the relationship between the generalized likelihood ratio test and backprojection method in synthetic aperture imaging," in Proc. SPIE Def., Secur. Sens. Conf., Orlando, FL, Apr. 2009, vol. 7335, pp. 73 350I-1-73 350I-10.

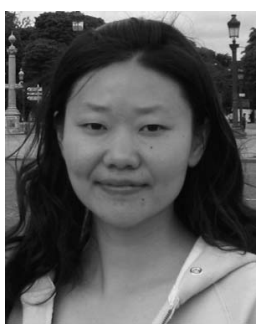

Ling Wang (M'07) received the B.S. degree in electrical engineering and the M.S. and Ph.D. degrees in information acquisition and processing from Nanjing University of Aeronautics and Astronautics, Nanjing, China, in 2000, 2003, and 2006, respectively.

Since 2003, she has been with Nanjing University of Aeronautics and Astronautics, where she is currently an Associate Professor with the Department of Information and Communication Engineering. From February 2008 to May 2009, she was a Postdoctoral Research Associate with the Department of Mathematical Sciences and the Department of Electrical, Computer, and Systems Engineering, Rensselaer Polytechnic Institute, Troy, NY. Her research interests include inverse scattering, wave-based imaging, radar imaging, and passive imaging.

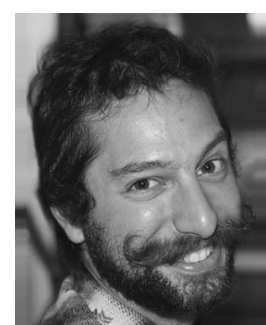

Can Evren Yarman received the B.Sc. degree in mathematics from the Middle East Technical University, Ankara, Turkey, the M.Sc. degree in biomedical science from Drexel University, Philadelphia, PA, and the M.Sc. degree in mathematics and the Ph.D. degree in electrical engineering from Rensselaer Polytechnic Institute, Troy, NY, in 2006.

From May 2006 to February 2007, he was a Postdoctorate with Rensselaer Polytechnic Institute. Currently, he is a Research Scientist with Houston Technology Center, WesternGeco-Schlumberger, Houston, TX. His main research interests include forward modeling and inverse problems in seismic, radar, and biomedical imaging.

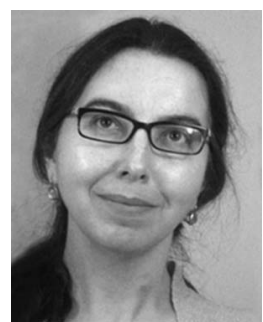

Birsen Yazıcı (SM'05) received B.S. degrees in electrical engineering and mathematics from Bogazici University, Istanbul, Turkey, in 1988 and the M.S. and $\mathrm{Ph} . \mathrm{D}$. degrees in mathematics and electrical engineering from Purdue University, West Lafayette, IN, in 1990 and 1994, respectively.

From September 1994 to 2000, she was a Research Engineer with the General Electric Company Global Research Center, Schenectady, NY. During her tenure in industry, she worked on radar, transportation, industrial, and medical imaging systems. From 2001 to June 2003, she was an Assistant Professor with the Department of Electrical and Computer Engineering, Drexel University, Philadelphia, PA. Since Fall 2003, she has been with Rensselaer Polytechnic Institute, Troy, NY, where she is currently an Associate Professor with the Department of Electrical, Computer, and Systems Engineering and the Department of Biomedical Engineering. She is the holder of 11 U.S. patents. Her research interests span the areas of statistical signal processing, inverse problems in imaging, image reconstruction, biomedical optics, radar, and X-ray imaging.

Dr. Yazici currently serves as an Associate Editor for the IEEE TRANSACTIONS ON IMAGE PROCESSING and SIAM Journal on Imaging Science. Her work on industrial systems received the 2nd best paper award in 1997 given by the IEEE TRANSACTIONS ON INDUSTRIal APPLiCATIONS. She was the recipient of the Rensselaer Polytechnic Institute 2007 School of Engineering Research Excellence Award. 